\title{
Immunization interventions to interrupt hepatitis $B$ virus mother-to-child transmission: a meta-analysis of randomized controlled trials
}

\author{
Hui Jin ${ }^{1,2+}$, Yueyuan Zhao ${ }^{1 \dagger}$, Zhaoying Tan ${ }^{3}$, Xuefeng Zhang ${ }^{3}$, Yaoyun Zhao ${ }^{1}$, Bei Wang ${ }^{1,2}$ and Pei Liu ${ }^{1,2^{*}}$
}

\begin{abstract}
Background: This study aimed to determine the clinical efficacy of various immune interventions on mother-to-child transmission (MTCT) of hepatitis B virus (HBV).

Methods: We retrieved different immune strategies on how to prevent MTCT reported in the literature from Chinese and English electronic databases from the viewpoint of intrauterine and extrauterine prevention. Relative risk (RR) and 95\% confidence interval (Cl) methods were used.

Results: Twenty-five articles on intrauterine prevention and 16 on extrauterine prevention were included in the analysis. Intrauterine prevention could reduce infants' HBV infection rate ( $R R=0.36,95 \% \mathrm{Cl}: 0.28-0.45)$ and increase their anti-hepatitis B surface-positive rate ( $\mathrm{RR}=2.42,95 \% \mathrm{Cl}: 1.46-4.01)$ at birth. Compared with passive immunization, passive-active immunization could reduce infants' HBV infection rate ( $\mathrm{RR}=0.66,95 \% \mathrm{Cl}: 0.52-0.84)$ at birth, even at more than 12 months of age ( $R R=0.54,95 \% \mathrm{Cl}: 0.42-0.69)$. Subgroup analysis demonstrated similar results except for pregnant women who were hepatitis B surface antigen-positive. Funnel plots and Egger's tests showed publication bias mainly in intrauterine prevention not in extrauterine one.

Conclusions: The long-term protective effect of pregnant women injected with hepatitis B immunoglobulin during pregnancy should be further validated by large-scale randomized trials. Newborns of pregnant women who carried HBV should undergo a passive-active immunization strategy.
\end{abstract}

Keywords: Hepatitis B immunoglobulin, Hepatitis B virus, Meta-analysis, Mother-to-child transmission

\section{Background}

Hepatitis B virus (HBV) infections are a global health problem [1]. Studies have shown that in neonates born to women who were hepatitis B surface antigen (HBsAg)positive, $10-20 \%$ were infected with $\mathrm{HBV}$, whereas those born to mothers who were HBsAg- and hepatitis B e antigen (HBeAg)-positive (double positive, DP), 90\% were infected with HBV [2]. Mother-to-child transmission (MTCT) greatly contributes to the persistence of the high number of HBV carriers because infections

\footnotetext{
*Correspondence: liupeiseu@163.com

${ }^{\dagger}$ Equal contributors

'Department of Epidemiology and Health Statistics, Southeast University, Nanjing, China

${ }^{2}$ Key Laboratory of Environmental Medicine Engineering, Ministry of Education, School of Public Health, Southeast University, Nanjing, China Full list of author information is available at the end of the article
}

occurring in neonates and in childhood result in a chronic HBV rate of $80-90 \%$ and $30-50 \%$, respectively [3].

Since the introduction of HBV vaccines (HBVac), the use of hepatitis B immunoglobulin (HBIG) and HBVac, termed passive-active immunization, has been efficient for preventing MTCT of HBV [4-6]. In the 1980s, studies showed that in newborns of HBsAg-positive mothers, the vertical transmission rate was reduced to $23 \%$ after vaccination with HBIG [7] and to 3-7\% after passive-active immunization [8]. Although a meta-analysis showed that the passive-active immunization was effective [5], Kenneth et al. [9] found that most of the studies were of low quality (e.g., lacking blinding and allocation concealment); few studies involved the effect of evaluating mothers who were HBsAg-positive and HBeAg-negative (single positive, SP).

Furthermore, $10-20 \%$ of newborns with HBsAgpositive mothers are still chronically infected with HBV, 
even after being vaccinated with HBIG and HBVac [10-12]. Wang et al. [13] and Zhang et al. [14] found that most immunization failures in newborns with DP mothers were due to intrauterine infection $[11,15]$. HBsAg does not cross the placenta, whereas HBeAg can cross the placenta and reach the fetus $[15,16]$. These studies suggested that intrauterine $\mathrm{HBV}$ infection had a close relationship with $\mathrm{HBeAg}$-positive mothers, preterm birth, and $\mathrm{HBV}$ in the placenta [11].

Several studies in China have suggested that there are protective effects, namely lower HBV infection rates or higher anti-hepatitis B surface (HBs) levels for newborns after their mothers were injected with HBIG during pregnancy [17-19] than those in a control group included in some meta-analyses $[20,21]$. However, Yuan et al. [22] found that there were no significant differences in newborns between vaccination and no vaccination with HBIG during pregnancy; they also suggested that HBV intrauterine transmission was not common [23-25]. Although previous meta-analysis to support the protective effects for newborns after their mothers were injected with HBIG during pregnancy, because they ignored the randomization group, or an imbalance of $\mathrm{HBeAg}$ infection status in pregnancy women could have potentially biased the results. Moreover, there was serious heterogeneity in these studies because of the quality of the studies included and the infection status of the mothers [26].

Therefore, based on system review and previous metaanalysis, this study aimed to update and again evaluate the effects of different immunization interventions, including mothers injected with HBIG during pregnancy and newborns injected with HBVac and/or HBIG to interrupt the MTCT of HBV.

\section{Methods}

\section{Search strategy}

We searched the Medline, EMBASE, Cochrane Library, China Biological Medicine Database, Chinese National Knowledge Infrastructure, and VIP Database for Chinese Technical Periodicals databases between January 1980 and December 2013 for relevant randomized controlled trials (RCTs) written in English and Chinese peer-reviewed literature. We used the terms "HBIG" (or "hepatitis B immunoglobulin") and "HBV" (or "hepatitis B virus") and "intrauterine" (or "ectopic" or "pregnant" or "pregnancy" or "mother" or "children" or "infant" or "newborn"). The bibliographies of the original studies, reviews, and relevant conference abstracts were manually searched.

\section{Inclusion and exclusion criteria}

The inclusion criteria designs or epidemiologic methods were RCTs. The subjects were HBsAg- and HBeAgpositive pregnant women or $\mathrm{HBsAg}$-positive pregnant women with a clear classification of HBeAg-positive and HBeAg-negative. The experimental and control groups were comparable, and one of the following comparisons was made. (1) In the experimental group, women in the third trimester of pregnancy were injected with HBIG; newborns were injected with HBIG and HBVac. In the control group, only newborns were injected with HBIG and HBVac. (2) In the experimental group, newborns were injected with HBIG and HBVac. In the control group, only newborns were injected with HBVac. (3) In the experimental group, women in the third trimester of pregnancy were injected with HBIG; newborns were injected with HBIG and HBVac. In the control group, only newborns were injected with HBVac. Subjects were asymptomatic HBsAg carriers during the study period.

Exclusion criteria were studies without a control group and studies with a control group without randomization. Only recent or detailed studies were chosen for repeated published studies.

\section{Data extraction and definitions of outcome}

Two researchers (HJ and YYZ) independently selected relevant studies and made a post-hoc assessment of methodological quality by means of the Cochrane library study quality evaluation tool [27]. The extracted data included the first author's name, year of publication, study method, treatment protocol, sample size, duration of follow-up, inclusion/exclusion criteria, and relevant outcome data.

With regard to outcome, we estimated the rate of infant HBV infection (HBsAg or HBV DNA) or protection (HBsAb) at various time points (within 24 hours of birth, at 7-12 months of age, and after 12 months of age) as the primary outcome. HBV intrauterine infection was defined as HBsAg and/or HBV DNA positivity in neonatal peripheral or umbilical blood within 24 hours of birth and before administration of active or passive immune prophylaxis. HBsAg-positive infections were classified as events (HBsAg-positive at any time $>1$ month of age) or as chronic (HBsAg-positive for 6 months).

\section{Quality assessment}

The quality of the studies was evaluated using the Cochrane Handbook for Systematic Reviews of Interventions (Additional file 1: Table S1), version 5.1.0, recommended standard: random sequence generation, allocation concealment, blinding, incomplete outcome data, selective reporting, and other biases. The risk of bias was regarded high in the presence of high bias in any domain, low if all key domains (except random sequence generation and allocation concealment) were of low bias, and unclear in all other cases. Two authors (HJ and ZT) independently assessed the risk of bias; when necessary, consensus was determined through help of a third author (PL). 


\section{Statistical analysis}

Statistical analysis was performed according to the intention-to-treat principle. The estimated pooled relative risk (relative ratio, RR) and 95\% confidence interval (95\% CI) were determined by the Mantel-Haenszel fixed-effects model, or the inverse variance randomeffects model. The heterogeneity test was used with the chi-squared test and $I^{2}$. An $I^{2}$ index of 25\%,50\%, and $75 \%$ indicated a low, moderate, and high degree of heterogeneity, respectively. $\mathrm{P}<0.10$ in the chi-squared test showed the existence of heterogeneity between studies.

Subgroup analysis included mothers with $\mathrm{HBeAg}$ status, the length of follow-up, and the quality of the included study. The Begg's [28] and Egger's [29] methods were used to check for publication bias. For all tests, $\mathrm{P} \leq$ 0.05 or $95 \%$ CIs not including " 1 " indicated statistical significance. The statistical analysis software used was RevMan 5.1.0 (Copenhagen: Nordic Cochrane Centre, The Cochrane Collaboration, 2011).

\section{Results}

Search results

Figure 1 is a flow chart of the included studies. The number of RCT studies on intrauterine and extrauterine prevention was 30 [22,30-58] and 24 [8,12,59-80], respectively. Among studies on intrauterine prevention, five were excluded because of duplicate publication and the remaining 25 (eight on mothers who were DP, 17 on those who were HBsAg- and/or HBeAg-positive), which were conducted in the mainland of China, were included. Among the studies on extrauterine prevention, eight studies were excluded because of duplicate publication, and the remaining 16 (13 on mothers who were $\mathrm{HBsAg-}$ and HBeAg-positive, three on those who were HBsAg- and/or HBeAg-positive) were included. The characteristics of included studies are shown in Tables 1 and 2.

\section{Quality assessment}

In intrauterine prevention (Figure 2A and Additional file 2: Figure S1A), four studies indicated that a random table was applied [22,32,35,54], whereas the remainder did not report the details of random-sequence generation. Allocation concealment was an undefined risk in the included studies because it was not reported. Four studies had a low attrition bias [22,32,36,51]; others were unclear. Performance and detection biases were low. Ten studies had high risk of reporting bias because of selective reporting.

In extrauterine prevention (Figure $2 \mathrm{~B}$ and Additional file 2: Figure S1B), two studies indicated that a random table was applied $[64,68]$, whereas the remainder did not report the details of random-sequence generation. All allocation concealment was unclear. Four studies had a low attrition bias $[12,61,67,80]$; others were unclear. Performance and detection biases were low.

\section{Meta-analysis results}

\section{Intrauterine and extrauterine prevention studies}

Table 3 and Figure $3 \mathrm{~A}$ show the comparison of immunization effects on newborns of HBV-infected women injected with HBIG and those without HBIG during pregnancy; they also show all of the newborns were

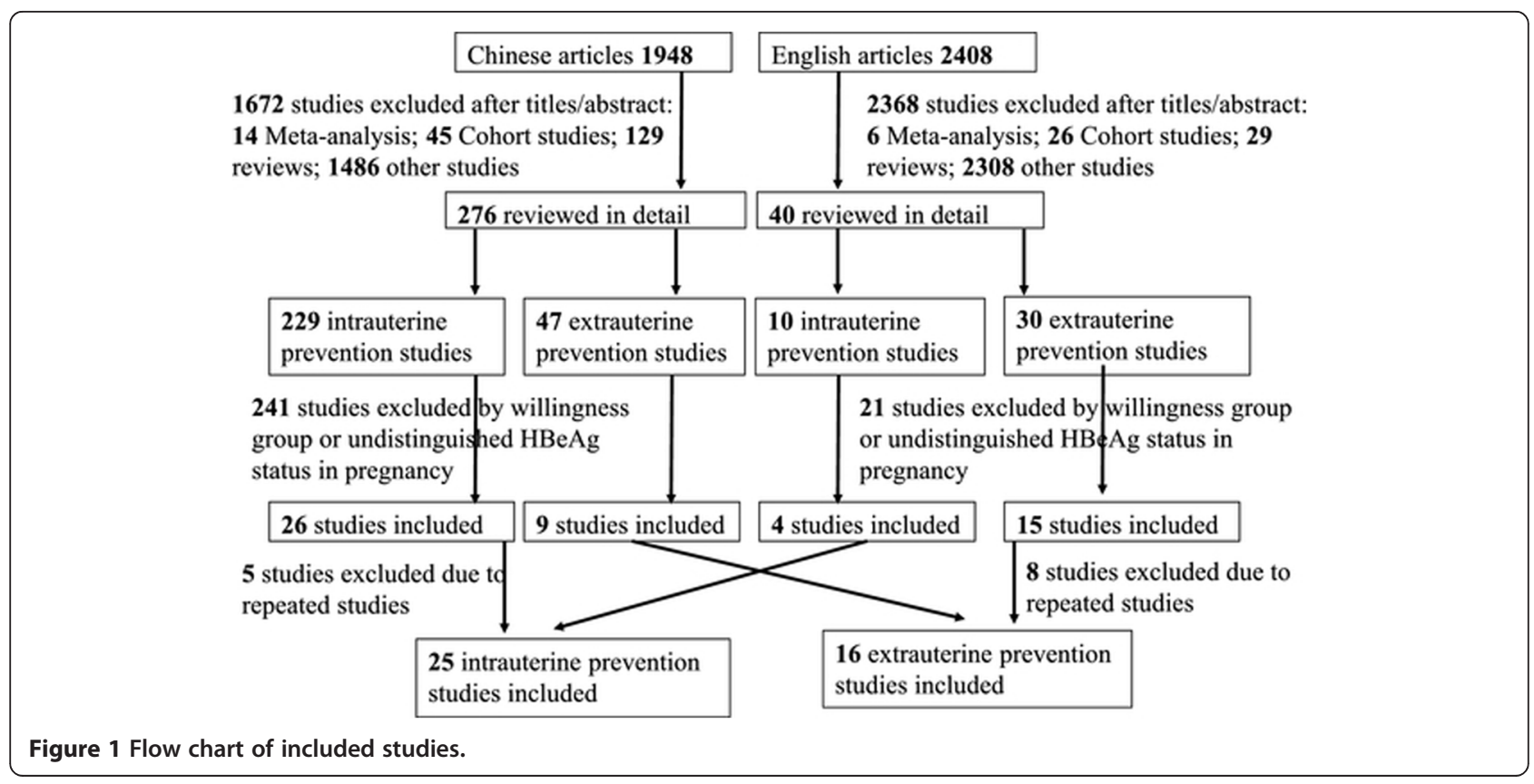


Table 1 Characteristics of intrauterine and extrauterine prevention for newborns born to HBsAg- and/or

HBeAg-positive women

\begin{tabular}{|c|c|c|c|c|c|c|c|c|c|c|c|}
\hline \multirow[t]{2}{*}{ Reference } & \multirow{2}{*}{$\begin{array}{l}\text { Mothers' } \\
\text { age (years) }\end{array}$} & \multirow[t]{2}{*}{$E^{1)}$} & \multicolumn{2}{|l|}{ Immune prophylaxis } & \multirow{2}{*}{$\begin{array}{l}\text { Sample } \\
\text { size }\end{array}$} & \multicolumn{2}{|l|}{ Newborn } & \multicolumn{2}{|c|}{ 7-12 month infant } & \multicolumn{2}{|c|}{$>12$ month child } \\
\hline & & & $\begin{array}{l}\text { Mother } \\
\text { (schedule/pregnancy month) }\end{array}$ & $\begin{array}{l}\text { Child } \\
\text { (schedule/infant month) }\end{array}$ & & HBsAg+ & $\overline{\mathrm{HBsAb+}}$ & $\mathrm{HBsAg}+$ & $\mathrm{HBsAb+}$ & $\mathrm{HBsAg}+$ & $\mathrm{HBsAb+}$ \\
\hline \multirow[t]{2}{*}{ Ji 2003 [30] } & $21-31$ & 1 & T: HBIG 200 IU $(7,8,9)$ & NR & $\mathrm{T}: 29$ & $\mathrm{~T}: 3$ & $\mathrm{~T}: 10$ & NR & NR & NR & NR \\
\hline & & & C: none & & $C: 31$ & C:5 & $C: 3$ & & & & \\
\hline Xu 2006 [31] & NR & 1 & T: HBIG 200 IU $(7,8,9)$ & NR & $\mathrm{T}: 30$ & $\mathrm{~T}: 7$ & NR & NR & NR & NR & NR \\
\hline Repeated [32] & & & C: none & & $C: 30$ & $C: 20$ & & & & & \\
\hline \multirow[t]{2}{*}{ Yuan 2006 [22] } & $20-33$ & 1 & T: HBIG 400 IU $(7,8,9)$ & T: HBIG $200 \mathrm{IU}(0)+\operatorname{RV} 5 \mathrm{ug}(0,1,6)$ & $\mathrm{T}: 118$ & $\mathrm{~T}: 27$ & $\mathrm{~T}: 0$ & $\mathrm{~T}: 13$ & $\mathrm{~T}: 101$ & NR & NR \\
\hline & & & C: Diluent & C: HBIG $200 \mathrm{IU}(0)+$ RV 5 ug $(0,1,6)$ & $C: 113$ & $C: 32$ & C:0 & $C: 17$ & C:112 & & \\
\hline \multirow[t]{3}{*}{ Chen 2007 [33] } & NR & 1 & T1: HBIG 200 IU $(7,8,9)$ & T1: HBIG $200 \mathrm{IU}(0,0.5)+$ RV 5 ug $(0,1,6)$ & $\mathrm{T} 1: 45$ & $\mathrm{~T} 1: 1$ & T1: 14 & $\mathrm{~T} 1: 1$ & $\mathrm{~T} 1: 33$ & NR & NR \\
\hline & & & T2: None & T2: HBIG $200 \mathrm{IU}(0,0.5)+$ RV 5 ug $(0,1,6)$ & $\mathrm{T} 2: 44$ & T2: No & T2: No & $\mathrm{T} 2: 3$ & T2: 35 & & \\
\hline & & & C:None & C: RV $5 u g(0,1,6)$ & $C: 49$ & C:13 & C: 4 & C: 13 & C: 32 & & \\
\hline \multirow[t]{3}{*}{ Sun 2007 [34] } & NR & 1 & T1: HBIG 200 IU $(7,8,9)$ & T1: HBIG $200 \mathrm{IU}(0,0.5)+\mathrm{V} 5 \mathrm{ug}(0,1,6)$ & $\mathrm{T} 1: 77$ & $\mathrm{~T} 1: 2$ & NR & $\mathrm{T} 1: 1$ & $\mathrm{~T} 1: 73$ & $\mathrm{~T} 1: 0$ & $\mathrm{~T} 1: 54$ \\
\hline & & & T2:None & T2: HBIG $200 \mathrm{IU}(0,0.5)+$ V 5 ug $(0,1,6)$ & $\mathrm{T} 2: 76$ & T2: 10 & & T2: 4 & $\mathrm{~T} 2: 70$ & $\mathrm{~T} 2: 1$ & T2: 50 \\
\hline & & & C: None & $C: \vee 5 u g(0,1,6)$ & $C: 70$ & C: 9 & & C: 8 & C: 58 & C: 4 & C: 30 \\
\hline \multirow[t]{2}{*}{ Wang 2007 [35] } & NR & 1 & T: HBIG 200 IU (4-9) & T: HBIG $200 \mathrm{IU}(0,0.5)+\mathrm{V} 10 \mathrm{ug}(1,2,7)$ & $\mathrm{T}: 32$ & T: 2 & NR & $\mathrm{T}: 2$ & NR & NR & NR \\
\hline & & & C: none & C: HBIG $200 \mathrm{IU}(0,0.5)+\mathrm{V} 10 \mathrm{ug}(1,2,7)$ & $C: 31$ & C: 11 & & C: 12 & & & \\
\hline \multirow[t]{2}{*}{ Yan 2009 [36] } & $22-35$ & 1 & T: HBIG 400 IU $(7,8,9)$ & T: HBIG $200 \mathrm{IU}(0,0.5)+\mathrm{RV} 10 \mathrm{ug}(0,1,6)$ & $T: 106$ & $\mathrm{~T}: 10$ & $\mathrm{~T}: 37$ & T: 9 & T: 82 & $\mathrm{~T}: 8$ & $\mathrm{~T}: 93$ \\
\hline & & & C: none & C: RV 10ug $(0,1,6)$ & C:98 & $C: 23$ & C: 9 & $C: 21$ & C: 46 & $C: 20$ & C: 69 \\
\hline \multirow[t]{2}{*}{ Cui 2011 [37] } & NR & 1 & T: HBIG 200 IU, 3 time & T: HBIG 100 IU, 2time + RV 5 ug, 0, 1,6 & $\mathrm{T}: 106$ & NR & NR & NR & NR & $\mathrm{T}: 5$ & $\mathrm{~T}: 96$ \\
\hline & & & C: none & C: RV 5 ug, $0,1,6$ & $C: 82$ & & & & & $C: 16$ & $C: 60$ \\
\hline \multirow[t]{2}{*}{ Zhu 1997 [38] } & NR & 1 & T: HBIG $200 \mathrm{IU}(7,8,9)$ & NR & $\mathrm{T}: 37$ & $\mathrm{~T}: 6$ & NR & NR & NR & NR & NR \\
\hline & & & & & $C: 32$ & C: 12 & & & & & \\
\hline \multirow[t]{2}{*}{ Repeated [39] } & & 2 & C: none & & $\mathrm{T}: 68$ & T: 0 & NR & NR & NR & NR & NR \\
\hline & & & & & $C: 70$ & $C: 3$ & & & & & \\
\hline \multirow[t]{4}{*}{ Jia 2001 [40] } & NR & 1 & T: HBIG $200 \mathrm{IU}(7,8,9)$ & NR & $\mathrm{T}: 15$ & T: 1 & NR & NR & NR & NR & NR \\
\hline & & & & & $C: 16$ & C: 7 & & & & & \\
\hline & & 2 & C: none & & $\mathrm{T}: 25$ & T: 0 & NR & NR & $N R$ & NR & NR \\
\hline & & & & & $C: 30$ & $C: 3$ & & & & & \\
\hline \multirow[t]{4}{*}{ Chi 2002 [41] } & NR & 1 & T: HBIG 200 IU $(7,8,9)$ & NR & $\mathrm{T}: 27$ & $\mathrm{~T}: 4$ & NR & NR & NR & NR & NR \\
\hline & & & & & $C: 29$ & $C: 10$ & & & & & \\
\hline & & 2 & C: none & & $\mathrm{T}: 42$ & T: 0 & $N R$ & NR & NR & NR & NR \\
\hline & & & & & $C: 43$ & $C: 2$ & & & & & \\
\hline
\end{tabular}


Table 1 Characteristics of intrauterine and extrauterine prevention for newborns born to HBsAg- and/or

HBeAg-positive women (Continued)

\begin{tabular}{|c|c|c|c|c|c|c|c|c|c|c|c|}
\hline \multirow[t]{4}{*}{ Chen2003 [42] } & \multirow[t]{4}{*}{ NR } & \multirow[t]{2}{*}{1} & \multirow[t]{2}{*}{ T: HBIG 200 IU $(7,8,9)$} & \multirow[t]{4}{*}{ NR } & T:18 & $\mathrm{T}: 2$ & \multirow[t]{2}{*}{ NR } & \multirow[t]{2}{*}{$N R$} & \multirow[t]{2}{*}{ NR } & \multirow[t]{2}{*}{ NR } & \multirow[t]{2}{*}{ NR } \\
\hline & & & & & $C: 15$ & $C: 6$ & & & & & \\
\hline & & \multirow[t]{2}{*}{2} & \multirow[t]{2}{*}{ C: none } & & $\mathrm{T}: 26$ & $\mathrm{~T}: 1$ & \multirow[t]{2}{*}{ NR } & \multirow[t]{2}{*}{ NR } & \multirow[t]{2}{*}{ NR } & \multirow[t]{2}{*}{ NR } & \multirow[t]{2}{*}{ NR } \\
\hline & & & & & $C: 20$ & $C: 2$ & & & & & \\
\hline \multirow[t]{2}{*}{ Han 2003 [43] } & \multirow[t]{4}{*}{ NR } & \multirow[t]{2}{*}{1} & \multirow[t]{2}{*}{ T: HBIG $200 \mathrm{IU}(7,8,9)$} & \multirow[t]{2}{*}{ T: HBIG $200 \mathrm{IU}(0,0.5)+$ V 5 ug $(1,2,7)$} & $\mathrm{T}: 83$ & $\mathrm{~T}: 21$ & \multirow[t]{2}{*}{ NR } & $\mathrm{T}: 5$ & \multirow[t]{2}{*}{ NR } & \multirow[t]{2}{*}{ NR } & \multirow[t]{2}{*}{ NR } \\
\hline & & & & & C:52 & $C: 23$ & & C: 7 & & & \\
\hline \multirow[t]{2}{*}{ Repeated [44] } & & 2 & C: None & $C:$ HBIG $200 \mathrm{IU}(0,0.5)+$ V 5 ug $(1,2,7)$ & $\mathrm{T}: 43$ & $\mathrm{~T}: 3$ & NR & $\mathrm{T}: 0$ & NR & NR & NR \\
\hline & & & & & C:38 & C: 9 & & C: 5 & & & \\
\hline Xing 2003 [45] & $22-38$ & 1 & T: HBIG $200 \mathrm{IU}(7,8,9)$ & NR & $\mathrm{T}: 16$ & $\mathrm{~T}: 2$ & NR & $N R$ & NR & NR & NR \\
\hline & & & & & $C: 15$ & $C: 6$ & & & & & \\
\hline Repeated [46] & & 2 & C: None & & $\mathrm{T}: 30$ & $\mathrm{~T}: 0$ & NR & $N R$ & NR & NR & NR \\
\hline & & & & & $C: 25$ & $C: 3$ & & & & & \\
\hline Zhu 2003 [47] & NR & 1 & T: HBIG 200-400 IU $(7,8,9)$ & $\mathrm{T}: \mathrm{HBIG} 100 \mathrm{IU}(0,0.5)+\mathrm{RV}$ & T:169 & $\mathrm{T}: 21$ & NR & NR & NR & NR & NR \\
\hline & & & & 3 ug( $(1,2,1)$ or PDV 30 ug $(1,2,1)$ & C:189 & C:49 & & & & & \\
\hline Repeated [48] & & 2 & C: none & C: $\mathrm{HBIG} 100 \mathrm{IU}(0,0.5)+\mathrm{RV}$ & $\mathrm{T}: 318$ & $\mathrm{~T}: 7$ & NR & $N R$ & $N R$ & NR & NR \\
\hline & & & & & C:304 & $C: 22$ & & & & & \\
\hline Chen 2006 [49] & NR & 1 & T: HBIG $200 \mathrm{IU}(7,8,9)$ & NR & T:16 & $\mathrm{T}: 4$ & NR & NR & NR & NR & NR \\
\hline & & & & & $\mathrm{C}: 14$ & C: 9 & & & & & \\
\hline & & 2 & C: none & & $\mathrm{T}: 34$ & $\mathrm{~T}: 1$ & NR & $N R$ & NR & NR & NR \\
\hline & & & & & C:36 & C: 5 & & & & & \\
\hline Yang 2006 [50] & NR & 1 & T: HBIG 200 IU (4-9) & NR & T:117 & $\mathrm{T}: 12$ & $\mathrm{~T}: 7$ & $N R$ & NR & NR & NR \\
\hline & & & C: None & & C:90 & C: 48 & $C: 0$ & & & & \\
\hline & & 2 & T: HBIG $200 \mathrm{IU}(7,8,9)$ & & $\mathrm{T}: 46$ & $\mathrm{~T}: 2$ & $\mathrm{~T}: 10$ & NR & NR & NR & NR \\
\hline & & & C: None & & C:32 & C:14 & C: 0 & & & & \\
\hline Yu 2006 [51] & NR & 1 & T1: HBIG 200-400 IU (7-10) & NR & $\mathrm{T} 1: 8$ & $\mathrm{~T} 1: 3$ & NR & NR & NR & NR & NR \\
\hline & & & & & $\mathrm{T} 2: 7$ & $\mathrm{~T} 2: 5$ & & & & & \\
\hline & & & & & $C: 8$ & C: 8 & & & & & \\
\hline & & 2 & T2: HBIG 200 IU $(7,8,9)$ & & $\mathrm{T} 1: 18$ & $\mathrm{~T} 1: 0$ & NR & $N R$ & NR & NR & NR \\
\hline & & & & & $\mathrm{T} 2: 22$ & $\mathrm{~T} 2: 0$ & & & & & \\
\hline & & & C: Diluent & & $C: 20$ & $C: 2$ & & & & & \\
\hline Ji 2007 [52] & NR & 1 & T: HBIG 200 IU $(7,8,9)$ & T: HBIG $200 \mathrm{IU}(0)+\mathrm{RV} 5 \mathrm{ug}(0,1,6)$ & $\mathrm{T}: 30$ & $\mathrm{~T}: 2$ & NR & $\mathrm{T}: 1$ & NR & NR & NR \\
\hline & & & & & $C: 26$ & $C: 10$ & & $C: 6$ & & & \\
\hline & & 2 & C: None & C: HBIG $200 \mathrm{IU}(0,0.5)+\mathrm{RV} 5 \mathrm{ug}(0,1,6)$ & $\mathrm{T}: 83$ & $\mathrm{~T}: 3$ & NR & $\mathrm{T}: 1$ & NR & NR & NR \\
\hline
\end{tabular}


Table 1 Characteristics of intrauterine and extrauterine prevention for newborns born to $\mathrm{HBsAg}$ - and/or

HBeAg-positive women (Continued)

\begin{tabular}{|c|c|c|c|c|c|c|c|c|c|c|c|}
\hline & & & & & $C: 84$ & C:5 & & C: 3 & & & \\
\hline \multirow[t]{4}{*}{ Liu 2007 [53] } & NR & 1 & T: HBIG 200 IU $(7,8,9)$ & T: HBIG $200 \mathrm{IU}(0,0.5)+\mathrm{RV} 10 \mathrm{ug}(0,1,6)$ & $\mathrm{T}: 12$ & $\mathrm{~T}: 1$ & $\mathrm{~T}: 4$ & $\mathrm{~T}: 0$ & $\mathrm{~T}: 10$ & NR & NR \\
\hline & & & & & $\mathrm{C}: 9$ & $C: 2$ & $C: 1$ & C: 2 & C: 4 & & \\
\hline & & 2 & C: None & C: HBIG $200 \mathrm{IU}(0,0.5)+\mathrm{RV} 10 \mathrm{ug}(0,1,6)$ & $\mathrm{T}: 31$ & $\mathrm{~T}: 1$ & $\mathrm{~T}: 12$ & $\mathrm{~T}: 0$ & $\mathrm{~T}: 24$ & NR & NR \\
\hline & & & & & $C: 34$ & C: 1 & C: 12 & $C: 1$ & C:25 & & \\
\hline \multirow[t]{4}{*}{ Wang 2008 [54] } & $20-33$ & 1 & T:HBIG 200 IU(5-9) & T: HBIG $200 \mathrm{IU}(0,0.5)+\mathrm{V}$ & $\mathrm{T}: 79$ & $\mathrm{~T}: 8$ & NR & $\mathrm{T}: 7$ & $N R$ & NR & NR \\
\hline & & & & & $C: 60$ & C: 19 & & C: 14 & & & \\
\hline & & 2 & C: None & $C: V$ & $\mathrm{~T}: 80$ & $\mathrm{~T}: 2$ & NR & $\mathrm{T}: 0$ & $N R$ & NR & NR \\
\hline & & & & & $C: 60$ & $C: 8$ & & C: 5 & & & \\
\hline \multirow[t]{4}{*}{ Zhao 2008 [55] } & $20-34$ & 1 & T: HBIG 200 IU $(7,8,9)$ & NR & $\mathrm{T}: 37$ & $\mathrm{~T}: 6$ & NR & NR & NR & NR & NR \\
\hline & & & & & $C: 32$ & $C: 12$ & & & & & \\
\hline & & 2 & C: none & & $\mathrm{T}: 66$ & $\mathrm{~T}: 0$ & NR & NR & $N R$ & NR & NR \\
\hline & & & & & $C: 69$ & $C: 3$ & & & & & \\
\hline \multirow[t]{3}{*}{ Liu 2009 [58] } & NR & 1 & T: HBIG 200 IU $(7,8,9)$ & NR & NR & NR & NR & NR & $N R$ & NR & NR \\
\hline & & 2 & C: none & & $\mathrm{T}: 100$ & $\mathrm{~T}: 1$ & NR & NR & $N R$ & NR & NR \\
\hline & & & & & $C: 120$ & $C: 4$ & & & & & \\
\hline \multirow[t]{6}{*}{ Yuan 2009 [57] } & $20-40$ & 1 & T1: HBIG 200 IU $(7,8,9)$ & T1: HBIG $200 \mathrm{IU}(0,0.5)+\mathrm{RV} 5 \mathrm{ug}(0,1,6)$ & $\mathrm{T} 1: 4$ & NR & NR & $\mathrm{T} 1: 0$ & $\mathrm{~T} 1: 13$ & NR & NR \\
\hline & & & & & $\mathrm{T} 2: 9$ & & & $\mathrm{~T} 2: 3$ & T2: 7 & & \\
\hline & & & T2: None & T2: HBIG $200 \mathrm{IU}(0,0.5)+$ RV 5 ug $(0,1,6)$ & $C: 13$ & & & C: 5 & C: 10 & & \\
\hline & & 2 & C: None & C: RV 5 ug $(0,1,6)$ & $\mathrm{T} 1: 23$ & NR & NR & $\mathrm{T} 1: 1$ & $\mathrm{~T} 1: 23$ & NR & NR \\
\hline & & & & & $\mathrm{T} 2: 13$ & & & $\mathrm{~T} 2: 2$ & $\mathrm{~T} 2: 12$ & & \\
\hline & & & & & $C: 13$ & & & C: 1 & C: 7 & & \\
\hline \multirow[t]{4}{*}{ Li 2013 [58] } & $24-35$ & 1 & T: HBIG 200 IU $(7,8,9)$ & T: HBIG $100 \mathrm{IU}, 6 \mathrm{~h}+\mathrm{RV} 10 \mathrm{ug}, 0,1,6$ & $\mathrm{~T}: 38$ & $\mathrm{~T}: 2$ & $\mathrm{~T}: 34$ & $\mathrm{~T}: 0$ & $\mathrm{~T}: 36$ & NR & NR \\
\hline & & & & & $C: 34$ & $C: 12$ & C:15 & $C: 11$ & $C: 15$ & & \\
\hline & & 2 & C: none & C: HBIG $100 \mathrm{IU}, 6 \mathrm{~h}+\mathrm{RV} 10 \mathrm{ug}, 0,1,6$ & $\mathrm{~T}: 14$ & $\mathrm{~T}: 0$ & $\mathrm{~T}: 13$ & $\mathrm{~T}: 0$ & $\mathrm{~T}: 14$ & NR & NR \\
\hline & & & & & $C: 28$ & $C: 3$ & C:20 & $C: 1$ & $C: 25$ & & \\
\hline
\end{tabular}

${ }^{1)} \mathrm{E}=\mathrm{HBeAg}, 1$ refers to pregnancy with $\mathrm{HBeAg}$ and $\mathrm{HBsAg}$ positivity; ${ }^{2}$ refers to pregnancy with $\mathrm{HBsAg}$ positivity and $\mathrm{HBeAg}$ negativity.

$\mathrm{T}$, experimental group; $\mathrm{C}$, control group.

$V$, vaccine; PDV, plasma-derived vaccine; RV, recombinant vaccine; $H B I G$, hepatitis $B$ immunoglobulin; NR, not reported. 
Table 2 Characteristics of extrauterine prevention alone for newborns born to HBsAg- and/or HBeAg-positive women

\begin{tabular}{|c|c|c|c|c|c|c|c|c|c|}
\hline \multirow[t]{2}{*}{ Reference } & \multirow[t]{2}{*}{$E^{1)}$} & \multirow{2}{*}{$\begin{array}{l}\text { Infant's Immune prophylaxis }{ }^{2)} \\
\text { (schedule/month ) }\end{array}$} & \multirow{2}{*}{$\begin{array}{l}\text { Sample } \\
\text { size (n) }\end{array}$} & \multicolumn{2}{|l|}{ Newborn } & \multicolumn{2}{|c|}{ 7-12month infant } & \multicolumn{2}{|c|}{$>12$ month child } \\
\hline & & & & HBsAg-pos & HBsAb-pos & HBsAg-pos & HBsAb-pos & HBsAg-pos & HBsAb-pos \\
\hline \multirow[t]{2}{*}{ Lo 1985 [59-61] } & 1 & T: HBIG50 IU(0) + PDV5ug & $\mathrm{T}: 36$ & NR & NR & $\mathrm{T}: 4$ & $\mathrm{~T}: 32$ & NR & NR \\
\hline & & $(0.5,1.5,2.5) ;$ C: PDV5ug $(0.5,1.5,2.5)$ & $C: 38$ & & & C: 9 & C:30 & & \\
\hline \multirow[t]{2}{*}{ Sha 1985 [62] } & 1 & T: HBIG $0.5 \mathrm{ml}(0)+$ PDV $20 \mathrm{ug}(0,1,2,12) ;$ & $\mathrm{T}: 19$ & $\mathrm{~T}: 13$ & T:18 & $\mathrm{T}: 4$ & $\mathrm{~T}: 10$ & NR & NR \\
\hline & & C: PDV 20 ug $(0,1,2,12)$ & $C: 10$ & $\mathrm{C}: 7$ & $\mathrm{C}: 0$ & $\mathrm{C}: 1$ & C:5 & & \\
\hline \multirow[t]{2}{*}{ Wu 1986 [63] } & 1 & $\mathrm{~T}: \mathrm{HBIG} 1 \mathrm{ml}(0)+$ PDV $20 \mathrm{ug}(1,2,3)$ & $\mathrm{T}: 13$ & $\mathrm{~T}: 1 ;$ & $N R$ & $N R$ & NR & NR & NR \\
\hline & & $C:$ PDV 20 ug $(1,2,3)$ & $\mathrm{C}: 6$ & $C: 0$ & & & & & \\
\hline \multirow[t]{2}{*}{ Farmer 1987 [64] } & 1 & T: HBIG 0.25 ml (25 IU/kg)(0,1.5), & $\mathrm{T}: 21$ & NR & NR & $\mathrm{T}: 3$ & $\mathrm{~T}: 17$ & NR & NR \\
\hline & & PDV5 ug $(0,1.5,6)$ C: PDV5 ug $(0,1.5,6)$ & $\mathrm{C}: 18$ & & & $C: 4$ & $C: 13$ & & \\
\hline \multirow[t]{2}{*}{ Theppisai 1987 [65] } & 1 & T: HBIG 200 IU(0) + PDV 10 ug $(0,1,6)$ & $\mathrm{T}: 27$ & NR & NR & $\mathrm{T}: 2$ & NR & NR & NR \\
\hline & & C: PDV 10 ug $(0,1,6)$ & $\mathrm{C}: 18$ & & & $C: 2$ & & & \\
\hline \multirow[t]{2}{*}{ Ip1 $989[8,66,67]$} & 1 & T: PDV3 ug $(0,1,2,6)+\operatorname{HBIG}(0)$ & $\mathrm{T}: 64$ & NR & NR & $\mathrm{T}: 8$ & NR & $\mathrm{T}: 9$ & $\mathrm{~T}: 47$ \\
\hline & & C: PDV3 ug $(0,1,2,6)$ & $C: 64$ & & & $C: 15$ & & $C: 15$ & C:52 \\
\hline \multirow[t]{2}{*}{ Assateerawatt 1993 [68] } & 1 & T:HBIG100IU(0) + RV20 ug $(0,1,2,12)$ & $\mathrm{T}: 30$ & $N R$ & NR & $\mathrm{T}: 1$ & $\mathrm{~T}: 25$ & $\mathrm{~T}: 1$ & $\mathrm{~T}: 24$ \\
\hline & & C: RV20 ug $(0,1,2,12)$ & $C: 30$ & & & $C: 2$ & $C: 22$ & $C: 3$ & $C: 21$ \\
\hline \multirow[t]{3}{*}{ Li 1994 [69] } & 1 & T: HBIG $200 \mathrm{IU}(0)+$ PDV $(0,1,6)$ & $\mathrm{T} 1: 20 ; \mathrm{C} 1: 22$ & $\mathrm{~T} 1: 7 ; \mathrm{C} 1: 7$ & $\mathrm{~T} 1: 18 ; \mathrm{C} 1: 3$ & $\mathrm{~T} 1: 1 ; \mathrm{C} 1: 3$ & $\mathrm{T1}: 16 ; \mathrm{C1}: 19$ & NR & NR \\
\hline & & $C: \operatorname{PDV}(1,2,3)$ & $\mathrm{T} 2: 20 ; \mathrm{C} 2: 21$ & T2:7; C2: 8; & T2:9; C2:2 & $\mathrm{T} 2: 7 ; \mathrm{C} 2: 7$ & $\mathrm{~T} 2: 17 ; \mathrm{C} 2: 11$ & & \\
\hline & & PDV including $10 \mathrm{ug}, 20 \mathrm{ug}$ and $30 \mathrm{ug}$ & T3:22; C3:21 & $\mathrm{T} 3: 7 ; \mathrm{C} 3: 7$ & T3:9; C3:2 & $\mathrm{T} 3: 1 ; \mathrm{C} 3: 2$ & T3:20; C3:20 & & \\
\hline \multirow[t]{2}{*}{ Zhao 1994 [70] } & 1 & $T: H B I G 60$ IU(0) + V 10 ug $(0,1,6)$ & $\mathrm{T}: 40$ & $\mathrm{~T}: 2$ & $\mathrm{~T}: 35$ & $\mathrm{~T}: 2$ & $\mathrm{~T}: 36$ & NR & NR \\
\hline & & $C: \vee 10$ ug $(0,1,6)$ & $C: 26$ & C:5 & C:9 & $C: 7$ & $C: 15$ & & \\
\hline \multirow[t]{2}{*}{ Kang 1995 [71] } & 1 & T: HBIG $200 \mathrm{IU}(0,1)+$ RV1 10 ug $(0,1,6)$ & $\mathrm{T}: 44$ & $N R$ & NR & $N R$ & NR & $\mathrm{T}: 0$ & $\mathrm{~T}: 43$ \\
\hline & & C: RV1 20 ug $(0,1,6)$ & $C: 41$ & & & & & C: 5 & C: 35 \\
\hline \multirow[t]{2}{*}{ Poovorawan 1997 [72-74] } & 1 & T: HBIG $100 \mathrm{IU}(0)+$ RV 10 ug $(0,1,6,60)$ & $\mathrm{T}: 64$ & $\mathrm{~T}: 1$ & NR & T: 0 & $\mathrm{~T}: 58$ & $\mathrm{~T}: 1$ & $\mathrm{~T}: 39$ \\
\hline & & C: RV 10 ug $(0,1,6,60)$ & C:63 & $C: 3$ & & $C: 3$ & C: 54 & $C: 3$ & $C: 35$ \\
\hline \multirow[t]{2}{*}{ Lin 2000 [75] } & 1 & $\mathrm{~T}: \mathrm{HBIG} 50 \mathrm{IU}(0)+\mathrm{RV} 10 \mathrm{ug}(0,1,6)$ & $\mathrm{T}: 31$ & $\mathrm{~T}: 4$ & $\mathrm{~T}: 26$ & $N R$ & NR & NR & NR \\
\hline & & $C:$ RV 10 ug $(0,1,6)$ & C:39 & $C: 2$ & C: 36 & & & & \\
\hline \multirow[t]{2}{*}{ Meng 2001 [76] } & 1 & $\mathrm{~T}: \mathrm{HBIG} 50 \mathrm{IU}(0)+\mathrm{RV} 10 \mathrm{ug}(0,1,6)$ & $\mathrm{T}: 50$ & NR & NR & NR & NR & $\mathrm{T}: 4$ & $\mathrm{~T}: 45$ \\
\hline & & $C:$ RV 10 ug $(0,1,6)$ & C:52 & & & & & $C: 7$ & $C: 43$ \\
\hline \multirow[t]{2}{*}{ Wang 2000-2001 [77,78] } & 1 & T: HBIG $100 \mathrm{IU}(0,1)+\operatorname{RV} 20 \mathrm{ug}(1,2,7)$ & $\mathrm{T}: 104 ; \mathrm{C}: 241$ & $\mathrm{~T}: 20 ; \mathrm{C}: 76$ & NR & $\mathrm{T}: 21 ; \mathrm{C}: 81$ & NR & T:26; C:96 & NR \\
\hline & 2 & C: PDV 20 ug $(0,1,6)$ & $\mathrm{T}: 157 ; \mathrm{C}: 122$ & $\mathrm{~T}: 19 ; \quad C: 25$ & NR & $\mathrm{T}: 22 ; \mathrm{C}: 26$ & NR & $\mathrm{T}: 28 ; \mathrm{C}: 35$ & NR \\
\hline
\end{tabular}


Table 2 Characteristics of extrauterine prevention alone for newborns born to HBsAg-and/or HBeAg-positive women (Continued)

\begin{tabular}{|c|c|c|c|c|c|c|c|c|c|}
\hline \multirow[t]{2}{*}{$\begin{array}{l}\text { Sehgal } 1992[79,80] \\
\end{array}$} & $1^{3)}$ & T: HBIG0.5 ml(0) + PDV10 ug $(0,1,2)$ & $\mathrm{T}: 7 ; \mathrm{C}: 7$ & NR & NR & $\mathrm{T}: 1 ; \mathrm{C}: 1$ & $\mathrm{~T}: 5 ; \mathrm{C}: 4$ & NR & NR \\
\hline & 2 & C: PDV10 ug $(0,1,2)$ & $\mathrm{T}: 17: \mathrm{C}: 14$ & NR & NR & $\mathrm{T}: 1 ; \mathrm{C}: 1$ & $\mathrm{~T}: 13 ; \mathrm{C}: 13$ & NR & NR \\
\hline \multirow[t]{2}{*}{ Xu 1995 [12] } & 1 & T: HBIG 250 IU(0) + PDV 20 ug $(0,1,6)$ & $\mathrm{T}: 11 ; \mathrm{C}: 31$ & NR & NR & $\mathrm{T}: 1 ; \mathrm{C}: 10$ & NR & $\mathrm{T}: 1 ; \mathrm{C}: 10$ & NR \\
\hline & 2 & C: PDV 20 ug $(0,1,6)$ & T:17; C:29 & NR & NR & $\mathrm{T}: 0 ; \mathrm{C}: 2$ & NR & $\mathrm{T}: 1 ; C: 2$ & NR \\
\hline
\end{tabular}

${ }^{1)} \mathrm{E}=\mathrm{HBeAg}, 1$ refers to pregnancy with $\mathrm{HBeAg}$ and HBsAg positivity; ${ }^{2}$ refers to pregnancy with HBsAg positivity and HBeAg negativity. ${ }^{2)}$ Vaccination schedule is filled in () by the unit of month.

T, experimental group; $C$, control group.

V, vaccine: PDV, plasma-derived vaccine; RV, recombinant vaccine; $\mathrm{HBIG}$, hepatitis B immunoglobulin: NR, not reported. ${ }^{35}$ Six newborns infected with HBV at birth were excluded owing to the absence of intervention. 


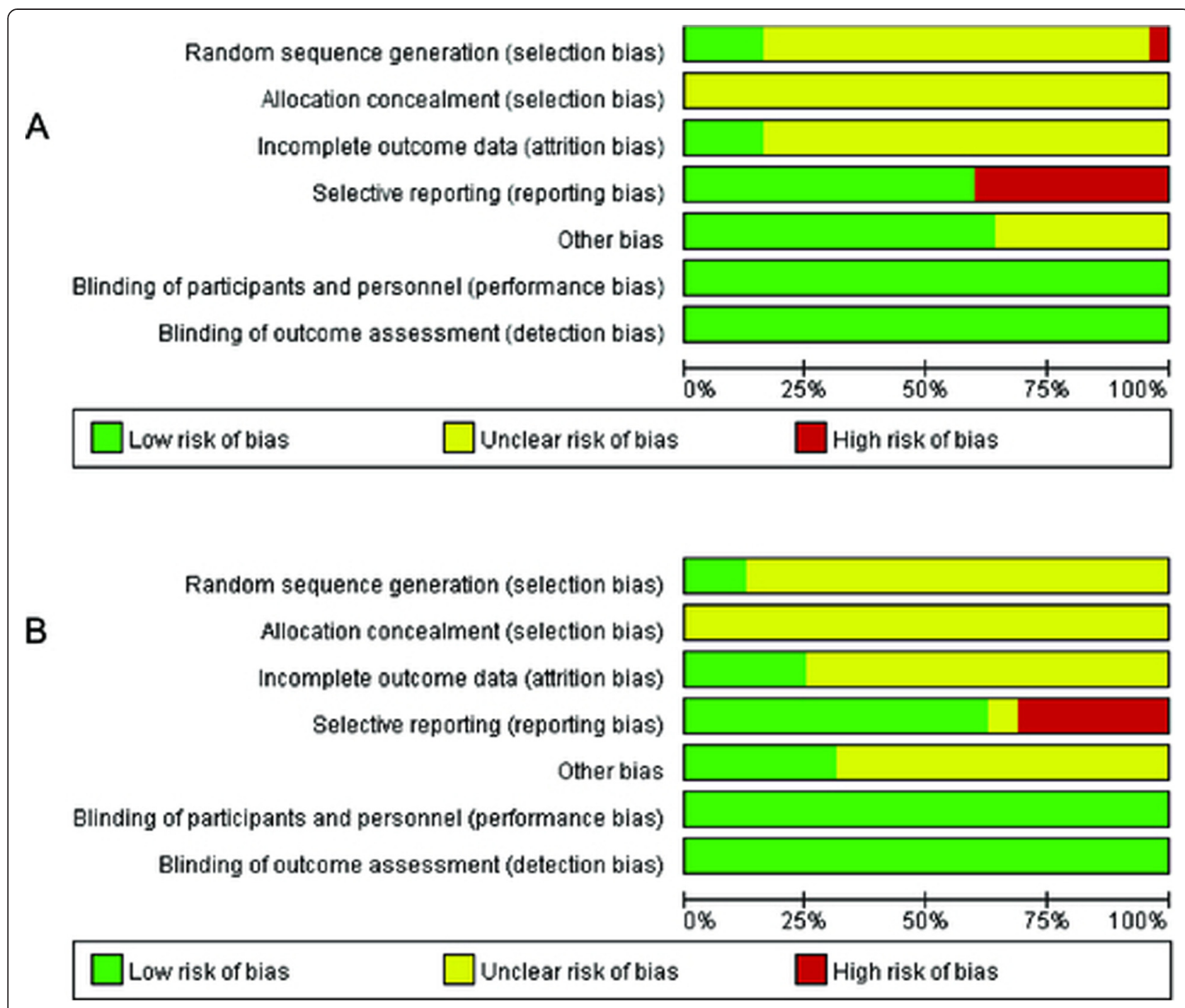

Figure $\mathbf{2}$ Risk of bias graph of included studies about intrauterine and extrauterine prevention. A. Intrauterine prevention. B. Extrauterine prevention.

injected with HBIG and HBVac. A total of 2192 newborns in the experimental group and 2082 in the control group at birth were included in 23 RCTs (Table 3 and Figure 3A). The meta-RR (95\% CI) comparing these two groups for newborn HBsAg infection rate was 0.36 $(0.28,0.45)$, and a medium level of heterogeneity was observed $\left(I^{2}=41 \%\right)$. There were 530 infants in the experimental group and 506 in the control group who had data on their serum HBsAg status at 7-12 months of age that were included in eight RCTs, with a meta-RR $(95 \% \mathrm{CI})$ of $0.34(0.22,0.53)\left(I^{2}=36 \%\right)$. However, only one RCT included those at more than 12 months of age (3 years), with a meta-RR $(95 \% \mathrm{CI})$ of $0.33(0.01,7.59)$. In subgroup analysis, there were similar protective effects as in these results, whether for maternal $\mathrm{HBeAg}$ status or for low risk and unclear bias (Table 3).
Meta-analysis showed that newborns in the experimental group had a higher amount of protective antibodies at birth, but not at the other time points (95\% CI including "1"), compared with the control group (Figure 3B). There were 556 newborns in the experimental group and 538 in the control group at birth, included in seven RCTs (Table 3 and Figure 3B). The meta-RR (95\% CI) comparing these two groups for newborn anti-HBs-positive rate at birth was $2.42(1.46,4.01)$, and a medium level of heterogeneity was observed $\left(I^{2}=64 \%\right)$. A total of 372 infants in the experimental group and 380 infants in the control group had data on their serum anti-HBs-positive status at 7-12 months of age were included in six RCTs, with a meta-RR $(95 \% \mathrm{CI})$ of $1.10(0.99,1.23)\left(I^{2}=68 \%\right)$. However, only one RCT included those who were older than 12 months of age (3 years), with a meta-RR $(95 \% \mathrm{CI})$ of $1.07(0.86,1.33)$. 
Table 3 Comparison of immunization effects on intrauterine and extrauterine prevention in newborns of HBsAg- and/or HBeAg-positive women ${ }^{1)}$

\begin{tabular}{|c|c|c|c|c|c|}
\hline Pregnancy infection status & Newborn infection status & Detective time & Number of included studies & Sample size & Meta-RR $(95 \% \mathrm{Cl})$ \\
\hline \multirow[t]{6}{*}{ Total (HBsAg+ and/or HBeAg+) } & \multirow[t]{3}{*}{$\mathrm{HBsAg}+$} & At birth & 23 & 4274 & $0.36(0.28,0.45)^{2)}$ \\
\hline & & 7-12 month & 8 & 1036 & $0.34(0.22,0.53)^{2)}$ \\
\hline & & $>12$ month & 1 & 153 & $0.33(0.01,7.95)^{2)}$ \\
\hline & \multirow[t]{3}{*}{$\mathrm{HBsAb}+$} & At birth & 7 & 1094 & $2.42(1.46,4.01)^{2)}$ \\
\hline & & 7-12 month & 6 & 757 & $1.12(1.00,1.24)^{2)}$ \\
\hline & & $>12$ month & 1 & 153 & $1.07(0.86,1.33)^{2)}$ \\
\hline \multirow[t]{6}{*}{ Subgroup (HBsAg+ and $\mathrm{HBeAg}+$ ) } & \multirow[t]{3}{*}{$\mathrm{HBsAg}+$} & At birth & 21 & 2159 & $0.40(0.32,0.51)^{2)}$ \\
\hline & & 7-12 month & 8 & 812 & $0.37(0.23,0.57)^{3)}$ \\
\hline & & $>12$ month & 1 & 153 & $0.33(0.01,7.95)^{2)}$ \\
\hline & \multirow[t]{3}{*}{$\mathrm{HBsAb+}$} & At birth & 7 & 909 & $3.05(2.19,4.25)^{2)}$ \\
\hline & & 7-12 month & 6 & 614 & $1.15(0.99,1.34)^{2)}$ \\
\hline & & $>12$ month & 1 & 153 & $\left.1.07(0.86,1.33)^{2}\right)$ \\
\hline \multirow[t]{4}{*}{ Subgroup (HBsAg+ and HBeAg-) } & \multirow[t]{2}{*}{$\mathrm{HBsAg}+$} & At birth & 15 & 1892 & $0.22(0.14,0.35)^{3)}$ \\
\hline & & 7-12 month & 4 & 224 & $0.22(0.06,0.84)^{3)}$ \\
\hline & \multirow[t]{2}{*}{$\mathrm{HBsAb+}$} & At birth & 3 & 185 & $1.31(0.82,2.10)^{3)}$ \\
\hline & & 7-12 month & 3 & 148 & $1.09(0.97,1.22)^{3)}$ \\
\hline \multirow[t]{6}{*}{ Subgroup (low+unclear bias) } & \multirow[t]{3}{*}{$\mathrm{HBsAg}+$} & At birth & 12 & 1945 & $0.35(0.22,0.54)^{2)}$ \\
\hline & & 7-12 month & 5 & 643 & $0.52(0.30,0.91)^{3)}$ \\
\hline & & $>12$ month & 1 & 153 & $0.33(0.01,7.95)^{3)}$ \\
\hline & \multirow[t]{3}{*}{$\mathrm{HBsAb+}$} & At birth & 6 & 980 & $3.02(1.48,6.15)^{2)}$ \\
\hline & & 7-12 month & 5 & 643 & $1.04(0.98,1.10)^{3)}$ \\
\hline & & $>12$ month & 1 & 153 & $1.07(0.86,1.33)^{3)}$ \\
\hline \multirow[t]{4}{*}{ Subgroup (high risk bias) } & \multirow[t]{2}{*}{$\mathrm{HBsAg}+$} & At birth & 11 & 2329 & $0.37(0.29,0.47)^{3)}$ \\
\hline & & 7-12 month & 3 & 393 & $0.18(0.08,0.39)^{3)}$ \\
\hline & \multirow[t]{2}{*}{$\mathrm{HBsAb+}$} & At birth & 1 & 114 & $1.60(1.26,2.03)^{3)}$ \\
\hline & & 7-12 month & 1 & 114 & $1.49(1.23,1.81)^{2)}$ \\
\hline
\end{tabular}

${ }^{1)}$ (Mother: HBIG/Infants: HBIG + vaccine) vs (Mother: none/Infants: HBIG + vaccine); ${ }^{2}$ Random effects model, inverse variance method; ${ }^{3)}$ Fixed effects model, Mantel-Haenszel method; values in boldface indicate statistical significance $(P<0.05)$.

In a subgroup analysis, there were similar protective effects as these results between the experimental and control groups, whether for maternal HBeAg status or for low risk and unclear bias (Table 3).

All of the Begg's tests, Egger's tests, and funnel plots revealed the existence of publication bias when comparing two groups for newborn HBsAg infection rate at birth and at 7-12 months of age (Figure 4A, B). Furthermore, the funnel plot was more skewed for the groups with HBV infection rate than for those with an antiHBs-positive rate.

\section{Extrauterine prevention studies}

Table 4 and Figure $3 \mathrm{C}$ show the effects of immunization between newborns injected with HBIG and HBVac and those who were vaccinated with only HBVac and whose mothers did receive HBIG injections during pregnancy. There were 490 newborns in the experimental group and 571 in the control group at birth in seven RCTs. The meta-RR (95\% CI) comparing these two groups for newborn HBsAg infection rate was 0.66 (0.52, 0.84); a low level of heterogeneity was observed $\left(I^{2}=28 \%\right)$. There were 677 infants in the experimental group and 776 in the control group with serum HBsAg status at 7-12 months of age that were included in 12 RCTs, with a meta-RR $(95 \% \mathrm{CI})$ of $0.54(0.42,0.69)\left(I^{2}=0 \%\right)$. Seven RCTs included data at more than 12 months of age, with a meta-RR $(95 \% \mathrm{CI})$ of $0.54(0.42,0.69)$. In subgroup analysis, there were similar protective effects as these results, whether for maternal HBeAg status or for low risk and unclear bias (Table 4).

Meta-analysis showed that newborns in the experimental group had a higher amount of protective antibodies at birth and at 7-12 months of age, but not at more than 12 months of age (95\% CI including "1"), compared with the control group (Figure 3D). There were 152 newborns 


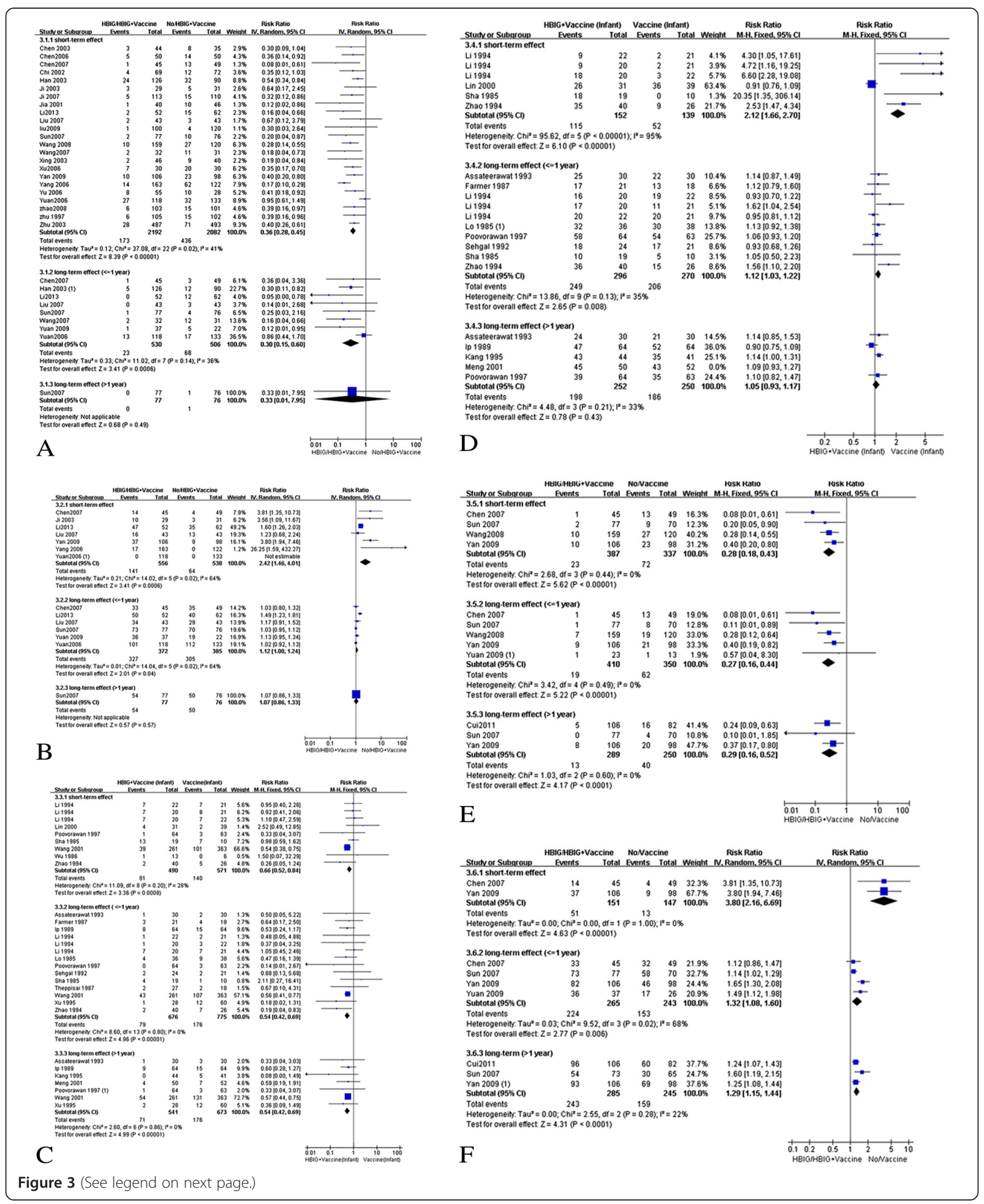


(See figure on previous page.)

Figure 3 Forest plot of HBV infection rates or the anti-HBs positive rate. (A) Forest plot of HBV infection rates of infants born to mothers with $\mathrm{HBsAg}$ and/or HBeAg positive for intrauterine and extrauterine prevention (mother: HBIG/infants: HBIG + vaccine vs mother: none/infants: HBUG + vaccine). (B) Forest plot of the anti-HBs positive rate of infants born to mothers with HBsAg and/or HBeAg positive for intrauterine and extrauterine prevention (mother: HBIG/infants: HBIG + vaccine vs mother: none/infants: HBUG + vaccine). (C) Forest plots of the HBV infection rate of infants born to mothers with HBsAg and/or HBeAg positive in extrauterine prevention. (D) Forest plot of the anti-HBs positive rate of infants born to mothers with $\mathrm{HBsAg}$ and/or HBeAg positive in extrauterine prevention. (E) Forest plot of the HBV infection positive rate of infants born to mothers with $\mathrm{HBsAg}$ and/or HBeAg positive for intrauterine and extrauterine prevention (mother: HBIG/infants: HBIG + vaccine vs mother: none/infants: vaccine). (F) Forest plot of the anti-HBs rate of infants born to mothers with $\mathrm{HBsAg}$ and/or HBeAg positive for intrauterine and extrauterine prevention (mother: HBIG/infants: HBIG + vaccine vs mother: none/infants: vaccine).

in the experimental group and 139 in the control group at birth that were included in four RCTs (Table 4 and Figure 3D). The meta-RR (95\% CI) comparing these two groups for newborn anti-HBs-positive rate at birth was $2.12(1.66,2.70)$; a higher level of heterogeneity was observed $\left(I^{2}=95 \%\right)$. There were 296 infants in the experimental group and 270 in the control group who had data on their serum anti-HBs-positive status at 7-12 months of age that were included in eight RCTs, with a meta-RR $(95 \% \mathrm{CI})$ of $1.12(1.03,1.22)\left(I^{2}=35 \%\right)$. Five RCTs included data at more than 12 months of age, with a meta-RR $(95 \% \mathrm{CI})$ of $1.06(0.96,1.16)$. In subgroup analysis, there were similar protective effects as these results between the experimental and control

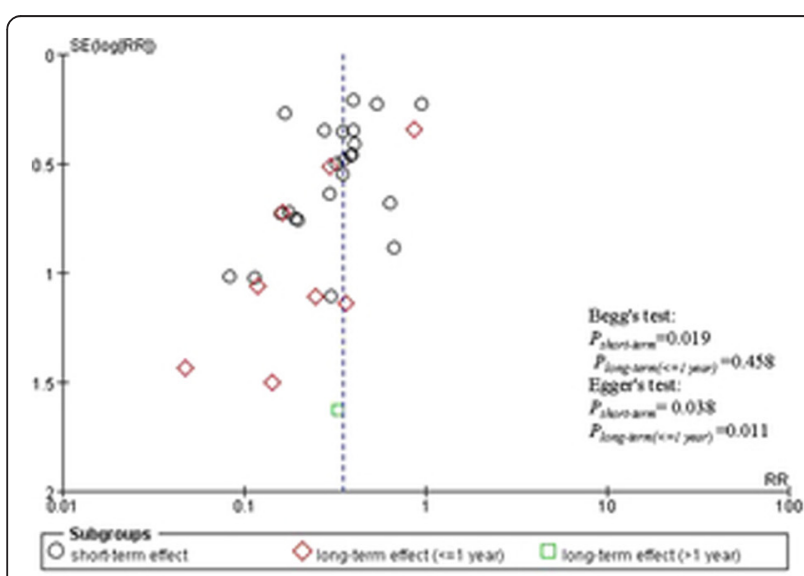

A

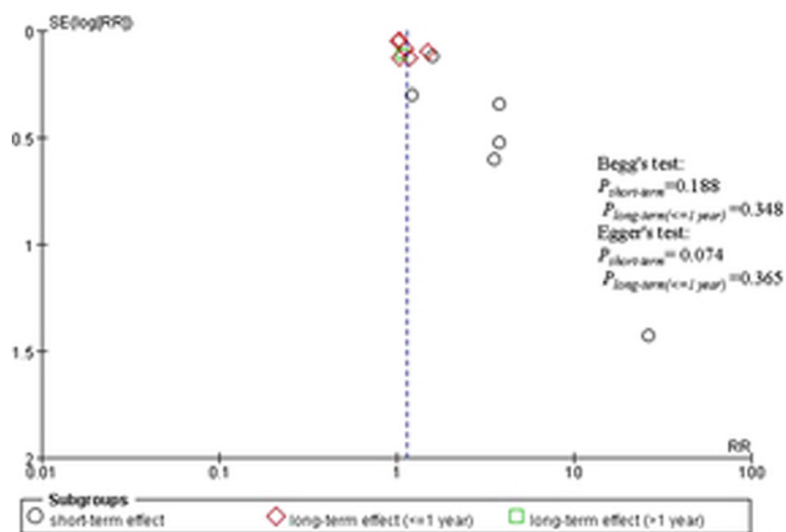

B

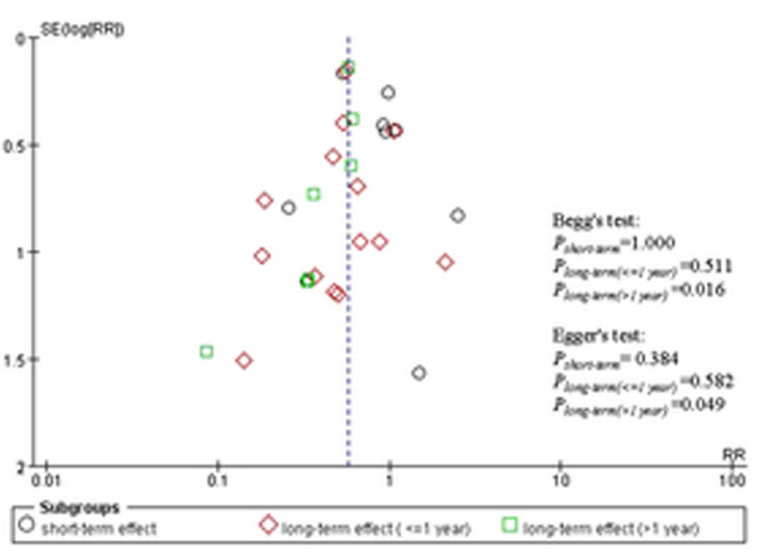

$\mathrm{C}$

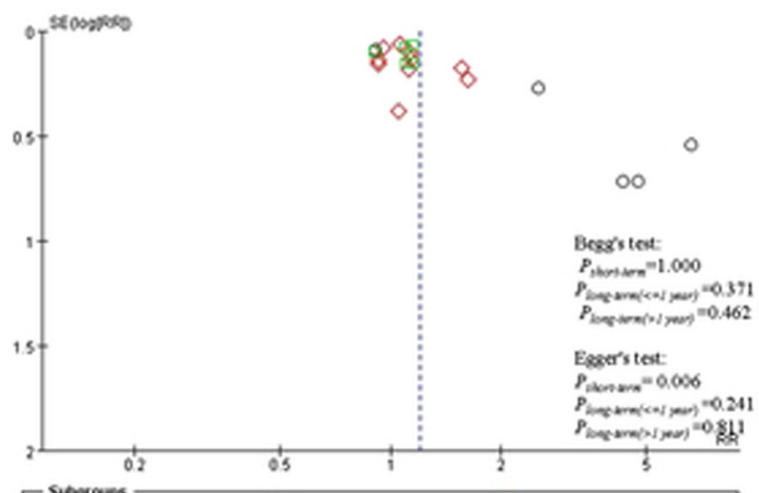

\begin{tabular}{|c|c|c|}
\hline O Suborows & 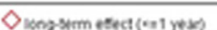 & metec \\
\hline
\end{tabular}

D

Figure 4 Funnel plot of HBV infection rate or anti-HBs positive rate. (A) Funnel plot of HBV infection rate of infants born to mothers with HBsAg and/or HBeAg positive for intrauterine and extrauterine prevention. (B) Funnel plot of the anti-HBs-positive rate of infants born to mothers with HBsAg and/or HBeAg positive for intrauterine and extrauterine prevention. (C) Funnel plot of HBV infection rate of infants born to mothers with HBsAg and/or HBeAg positive for intrauterine and/or extrauterine prevention. (D) Funnel plot of the anti-HBs-positive rate of infants born to mothers with HBsAg and/or HBeAg positive for intrauterine and/or extrauterine prevention. 
Table 4 Comparison of immunization effects on extrauterine prevention alone for newborns of $\mathrm{HBsAg}$ - and/or HBeAg-positive women ${ }^{\text {\& }}$

\begin{tabular}{|c|c|c|c|c|c|}
\hline Pregnancy infection status & Newborn infection status & Detective time & Number of included studies & Sample size & Meta-RR $(95 \% \mathrm{Cl})$ \\
\hline \multirow[t]{6}{*}{ Total (HBsAg+ and/or $\mathrm{HBeAg}+)$} & \multirow[t]{3}{*}{$\mathrm{HBsAg}+$} & At birth & 7 & 1061 & $0.66(0.52,0.84)^{3)}$ \\
\hline & & 7-12 month & 12 & 1451 & $0.54(0.42,0.69)^{3)}$ \\
\hline & & $>12$ month & 7 & 1214 & $0.54(0.42,0.69)^{3)}$ \\
\hline & \multirow[t]{3}{*}{$\mathrm{HBsAb+}$} & At birth & 4 & 291 & $2.12(1.66,2.70)^{3)}$ \\
\hline & & 7-12 month & 8 & 566 & $1.12(1.03,1.22)^{3)}$ \\
\hline & & $>12$ month & 5 & 502 & $1.06(0.96,1.16)^{3)}$ \\
\hline \multirow[t]{6}{*}{ Subgroup (HBsAg+ and $\mathrm{HBeAg}+$ ) } & \multirow[t]{3}{*}{$\mathrm{HBsAg}+$} & At birth & 7 & 782 & $0.75(0.57,0.99)^{3)}$ \\
\hline & & 7-12 month & 12 & 1095 & $0.56(0.42,0.75)^{3)}$ \\
\hline & & $>12$ month & 7 & 889 & $0.55(0.41,0.75)^{3)}$ \\
\hline & \multirow[t]{3}{*}{$\mathrm{HBsAb+}$} & At birth & 4 & 291 & $3.25(1.35,7.83)^{2)}$ \\
\hline & & 7-12 month & 9 & 443 & $1.14(1.05,1.24)^{3)}$ \\
\hline & & $>12$ month & 5 & 502 & $1.06(0.96,1.16)^{3)}$ \\
\hline \multirow[t]{3}{*}{ Subgroup (HBsAg+ and HBeAg-) } & \multirow[t]{3}{*}{$\mathrm{HBsAg}+$} & At birth & 1 & 279 & $0.59(0.34,1.02)^{3)}$ \\
\hline & & 7-12 month & 3 & 356 & $0.64(0.39,1.06)^{3)}$ \\
\hline & & $>12$ month & 2 & 325 & $0.63(0.41,0.97)^{3)}$ \\
\hline \multirow[t]{6}{*}{ Subgroup (low + unclear bias) } & \multirow[t]{3}{*}{$\mathrm{HBsAg}+$} & At birth & 4 & 348 & $0.82(0.58,1.18)^{3)}$ \\
\hline & & 7-12 month & 10 & 739 & $0.56(0.38,0.82)^{3)}$ \\
\hline & & $>12$ month & 4 & 400 & $0.43(0.23,0.82)^{3)}$ \\
\hline & \multirow[t]{3}{*}{ HBsAb+ } & At birth & 3 & 221 & $4.21(2.70,6.57)^{3)}$ \\
\hline & & 7-12 month & 8 & 566 & $1.12(1.03,1.22)^{3)}$ \\
\hline & & $>12$ month & 4 & 400 & $1.05(0.93,1.17)^{3)}$ \\
\hline \multirow[t]{6}{*}{ Subgroup (high risk bias) } & \multirow[t]{3}{*}{$\mathrm{HBsAg}+$} & At birth & 3 & 713 & $0.58(0.42,0.81)^{3)}$ \\
\hline & & 7-12 month & 2 & 712 & $0.53(0.39,0.72)^{3)}$ \\
\hline & & $>12$ month & 3 & 814 & $0.56(0.43,0.73)^{3)}$ \\
\hline & \multirow[t]{3}{*}{$\mathrm{HBsAb+}$} & At birth & 1 & 70 & $0.91(0.76,1.09)$ \\
\hline & & 7-12 month & - & - & - \\
\hline & & $>12$ month & 1 & 102 & $1.09(0.93,1.27)$ \\
\hline
\end{tabular}

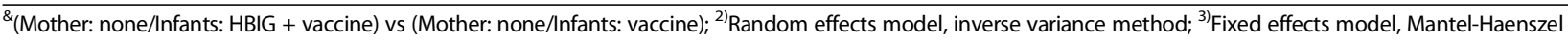
method; values in boldface indicate statistical significance $(P<0.05)$.

groups for pregnant women with DP that included studies with low risk and unclear bias (Table 4). However, there was lack of data about anti-HBs-positive status when comparing these two groups for newborns of women with SP.

Egger's test revealed the existence of publication bias when comparing two groups for newborn HBsAg infection rate at more than 12 months of age and for newborn anti-HBs-positive rate at birth (Figure 4C,D). The funnel plots show similar results but with a more skewed shape.

\section{Other prevention studies}

Figure 3E and Figure 3F show a comparison of the effects of immunization between HBV-infected mothers who received HBIG during pregnancy and newborns that received $\mathrm{HBIG}$ and $\mathrm{HBVac}$ versus newborns that received HBVac. Meta-analysis showed that newborns in the experimental group had a lower infection rate and a higher amount of protective antibodies at each time point than did the control group. Publication bias was not shown because of the low number of included studies.

\section{Safety analysis}

No adverse events-such as fever, rigor, skin rash, inflammation, scleroma at the locally injected area, impairment of renal function, or other discomforts-were found in any of the included studies.

\section{Discussion}

In this study, meta-analysis was used to investigate the clinical effects of different immunization strategies on interrupting MTCT of HBV. The main findings of our study follow. 
First, our study found that multiple small doses of intramuscular HBIG injection in HBV-carrying mothers during the third trimester of pregnancy could reduce infants' HBV infection rate and increase their anti-HBspositive rate at birth. However, there was no statistical significance in the anti-HBs-positive rate of newborns at more than 7 months of age between the experimental and control groups. Subgroup analysis, such as for DP pregnant women and for the studies without a high risk of bias, showed similar results. The possible mechanism for this is that HBIG administration produces shortterm effects, whereas passive HBVac immunization has long-term effects. Furthermore, only one RCT [34] with 3 years' follow-up supported this view; its meta-RR (95\% CI) was $0.33(0.01,7.59)$. Recently, other studies $[17,19]$ have indicated that prenatal HBIG vaccination is effective and improves the immune response for DP pregnant women. Shi et al. [20] and $\mathrm{Xu}$ et al. [21] used metaanalyses to show the same viewpoint as these other studies, but Yuan et al. [22] proposed the opposite view. They found that there were no significant differences in newborns between those vaccinated and not vaccinated with HBIG during pregnancy. Our study suggested that multiple small doses of intramuscular HBIG injection during the third trimester of pregnancy still need to be proven by RCTs; this was based on the following considerations. Most of the included studies had an unclear or higher risk of bias and there was clearly publication bias. Extensive HBIG vaccination might lead to immune resistance to HBV strains, which potentially results in the HBVac being ineffective. Additionally, it is possible to produce antigen-antibody immune complexes in vivo in pregnant women injected with HBIG that threaten maternal and fetal health.

Second, in pregnant women who carry HBV, passiveactive immunization can be efficient for preventing MTCT of HBV [5], especially for DP pregnant women. Notably, most of the RCT studies were carried out before 2000 because immunization strategies were recommended by many countries at that same time. Additionally, many included studies were of low quality, such as a lack of blinding and allocation concealment. Fortunately, subgroup analysis and funnel plots supported the previously mentioned conclusion. There are few studies on SP pregnant women in the study because some quasi-RCT studies without random allocation or with allocation according to willingness were excluded. In SP pregnant women, subgroup analysis showed a lower HBsAg infection rate for newborns who received HBIG and HBVac than those who had just HBVac at more than 12 months of age. This suggests that newborns of SP pregnant women should receive passive-active immunization [81].

Third, more attention should be paid to some neglected issues in the application of clinical trials in vertical MTCT of HBV. Considering the feasibility of trials, many researchers used the method of allocation according to patient willingness [82], not random allocation. Whether pregnant women are willing to be injected with HBIG is dependent on factors such as economic condition, educational level, and HBeAg status. Imbalance of these factors between groups would bias the results. Furthermore, most-except for a few [22,31,54] —of the included studies in our meta-analysis did not describe how to randomly divide study subjects into groups. In addition, blinding and control choice need to be carefully considered; therefore, researchers should increase their cooperation with statisticians and epidemiologists and carefully design clinical trials with them under the guide of the CONSORT criteria before starting a trial.

Our study had the following advantages. (1) Multiple immunization strategies were used to comprehensively investigate, evaluate, and compare strategies. These methods were helpful for minimizing the effect of bias and for improving the accuracy of the study. (2) HBeAg positivity had a close relationship with HBV DNA level. Having access to $\mathrm{HBeAg}$ infection status was helpful for controlling bias for evaluating immunization strategy [81]. Wen et al. [83] found that offspring of HBeAgpositive mothers were more likely to be infected and to become chronic carriers than those of HBeAg-negative mothers. This was most likely attributable to the difference in maternal viral load or HBV DNA level. (3) Unlike previous meta-analyses [20,21], the quality of our studies was evaluated using the Cochrane Handbook for Systematic Reviews of Interventions, version 5.1.0, recommended standard. Subgroup analysis of non-highrisk bias facilitated improved appraisal of evidence and led to better health care. In addition, including more studies increased the statistical efficacy.

Our study had the following limitations. (1) Different from extrauterine prevention, intrauterine prevention included these RCTs all from Chinese studies. This limits our results in terms of how to generalize them to other countries. (2) The meta-analysis of SP pregnant women was based on subgroup analysis or non-RCT studies; therefore, these results need to be verified by further RCT studies. (3) Long-term effects in the RCTs were difficult to obtain, especially for certain time points. (4) A lack of sufficient information can bias the results (e.g., mode of delivery, maternal HBV change, laboratory technology, patient HBV DNA level). If umbilical blood is contaminated by maternal body fluids, the error diagnosis of newborn HBV infection could misinform the final results. In addition, it is essential to consider cost-effectiveness analysis, such as the relationship between the cost of multiple small doses of HBIG and protective effects, to evaluate different immunization interventions. 


\section{Conclusions}

Although our meta-analysis shows a protective effect of HBIG vaccination of women during the third trimester of pregnancy, this should be further validated by longterm, large-scale randomized trials. In addition, newborns of both DP women and SP women should receive passive-active immunization.

\section{Additional files}

Additional file 1: Table S1. PRISMA 2009 Checklist.

Additional file 2: Figure S1. Risk of bias summary for each included study. A. Intrauterine prevention. B. Extrauterine prevention.

\section{Competing interests}

The authors declare that they have no competing interest.

\section{Authors' contributions}

$H J, Y Y Z, Z T, Y Y Z$, and BW were responsible for literature search and retrieving data; HJ, XZ, BW, and PL were responsible for design and concept of the manuscript; all authors were responsible for the analysis and writing of the manuscript. All authors read and approved the final manuscript.

\section{Acknowledgments}

The authors were supported by the National Science and Technology Major Project of China (2009ZX10004-904), the Social Development Fund of Jiangsu Province (7725000014), and the Department of Health of Jiangsu Province (Y2012070). We acknowledge Qian Gao for her assistance with identifying and screening the abstracts of studies obtained from the searched databases.

\section{Author details}

${ }^{1}$ Department of Epidemiology and Health Statistics, Southeast University, Nanjing, China. ${ }^{2}$ Key Laboratory of Environmental Medicine Engineering, Ministry of Education, School of Public Health, Southeast University, Nanjing, China. ${ }^{3}$ Jiangsu Provincial Centre for Disease Control and Prevention, Nanjing, China.

Received: 18 September 2014 Accepted: 7 December 2014

Published online: 20 December 2014

\section{References}

1. Gambarin-Gelwan M: Hepatitis B in pregnancy. Clin Liver Dis 2007, 11:945-963.

2. Stevens CE, Toy PT, Tong MJ, Taylor PE, Vyas GN, Nair PV: Perinatal hepatitis $B$ virus transmission in the United States. Prevention by passive-active immunization. JAMA 1985, 253:1740-1745.

3. Hyams KC: Risks of chronicity following acute hepatitis B virus infection: a review. Clin Infect Dis 1995, 20:992-1000.

4. Beasley RP: Rocks along the road to the control of HBV and HCC. Ann Epidemiol 2009, 9:231-234.

5. Lee C, Gong Y, Brok J, Boxall EH, Gluud C: Effect of hepatitis B immunisation in newborn infants of mothers positive for hepatitis $B$ surface antigen: systematic review and meta-analysis. BMJ 2006, 332:328-336.

6. Shapiro CN: Epidemiology of hepatitis B. Pediatr Infect Dis J 1993, 12:433-437.

7. Beasley RP, Hwang LY, Lin CC, Stevens CE, Wang KY, Sun TS: Hepatitis B immune globulin (HBIG) efficacy in the interruption of perinatal transmission of hepatitis B virus carrier state. Initial report of a randomised double-blind placebo-controlled trial. Lancet 1981, 2:388-393.

8. Wong VC, Ip HM, Reesink HW, Lelie PN, Reerink-Brongers EE, Yeung CY, Ma HK: Prevention of the HBsAg carrier state in newborn infants of mothers who are chronic carriers of $\mathrm{HBsAg}$ and $\mathrm{HBeAg}$ by administration of hepatitis-B vaccine and hepatitis-B immunoglobulin. Double-blind randomised placebo-controlled study. Lancet 1984, 1:921-926.

9. Lin K, Vickery J: Screening for hepatitis B virus infection in pregnant women: evidence for the U.S. Preventive Services Task Force reaffirmation recommendation statement. Ann Intern Med 2009, 150:874-876.
10. Wang JS, Zhu QR, Wang XH: Breastfeeding does not pose any additional risk of immunoprophylaxis failure on infants of HBV carrier mothers. Int J Clin Pract 2003, 57:100-102.

11. Xu DZ, Yan YP, Choi BC, Xu JQ, Men K, Zhang JX, Liu ZH, Wang FS: Risk factors and mechanism of transplacental transmission of hepatitis $B$ virus: a case-control study. J Med Virol 2002, 67:20-26.

12. Xu ZY, Duan SC, Margolis HS, Purcell RH, Ou-Yang PY, Coleman PJ: Longterm efficacy of active postexposure immunization of infants for prevention of hepatitis B virus infection. United States-People's Republic of China Study Group on Hepatitis B. J Infect Dis 1995, 171:54-60.

13. Wang JS, Chen H, Zhu QR: Transformation of hepatitis B serologic markers in babies born to hepatitis B surface antigen positive mothers. World J Gastroenterol 2005, 11:3582-3585.

14. Zhang SL, Yue YF, Bai GQ, Shi L, Jiang H: Mechanism of intrauterine infection of hepatitis B virus. World J Gastroenterol 2004, 10:437-438.

15. Milich D, Liang TJ: Exploring the biological basis of hepatitis $B$ e antigen in hepatitis B virus infection. Hepatol 2003, 38:1075-1086.

16. Wang JS, Zhu QR: Infection of the fetus with hepatitis $B$ e antigen via the placenta. Lancet 2000, 355:989.

17. Li XM, Shi MF, Yang YB, Shi ZJ, Hou HY, Shen HM, Teng BQ: Effect of hepatitis B immunoglobulin on interruption of HBV intrauterine infection. World J Gastroenterol 2004, 10:3215-3217.

18. Li XM, Yang YB, Hou HY, Shi ZJ, Shen HM, Teng BQ, Li AM, Shi MF, Zou L: Interruption of HBV intrauterine transmission: a clinical study. World J Gastroenterol 2003, 9:1501-1503.

19. Xiao XM, Li AZ, Chen X, Zhu YK, Miao J: Prevention of vertical hepatitis $B$ transmission by hepatitis $B$ immunoglobulin in the third trimester of pregnancy. Int J Gynaecol Obstet 2007, 96:167-170.

20. Shi Z, Li X, Ma L, Yang Y: Hepatitis B immunoglobulin injection in pregnancy to interrupt hepatitis $B$ virus mother-to-child transmission-a meta-analysis. Int J Infect Dis 2010, 14:e622-e634.

21. Xu H, Zeng T, Liu JY, Lei Y, Zhong S, Sheng YJ, Zhou Z, Ren H: Measures to Reduce Mother-to-Child Transmission of Hepatitis B Virus in China: A Meta-Analysis. Dig Dis Sci 2013. doi:10.1007/s10620-013-2918-0

22. Yuan J, Lin J, Xu A, Li H, Hu B, Chen J, Yao J, Dong H, Jiang M: Antepartum immunoprophylaxis of three doses of hepatitis $B$ immunoglobulin is not effective: a single-centre randomized study. J Viral Hepat 2006, 13:597-604

23. Ranger-Rogez S, Denis F: Hepatitis B mother-to-child transmission. Expert Rev Anti Infect Ther 2004, 2:133-145.

24. Rumi MA, Begum K, Hassan MS, Hasan SM, Azam MG, Hasan KN, Shirin M, Khan AK: Detection of hepatitis B surface antigen in pregnant women attending a public hospital for delivery: implication for vaccination strategy in Bangladesh. Am J Trop Med Hyg 1998, 59:318-322.

25. Poovorawan $Y$, Chongsrisawat $V$, Theamboonlers A, Vimolkej L, Yano M: Is there evidence for intrauterine $\mathrm{HBV}$ infection in newborns of hepatitis B carrier mothers? Southeast Asian J Trop Med Public Health 1997, 28:365-369.

26. Jonas MM: Hepatitis B and pregnancy: an underestimated issue. Liver Int 2009, 29(Suppl 1):133-139.

27. Julian H, Douglas GA, Peter CG, Juni P, Moher D, Oxman AD, Savovic J, Schulz KF, Weeks L, Steme JA: The Cochrane Collaboration's tool for assessing risk of bias in randomised trials. BMJ 2011, 343:d5928.

28. Begg CB, Mazumdar M: Operating characteristics of a rank correlation test for publication bias. Biometrics 1994, 50:1088-1101.

29. Egger M, Davey SG, Schneider M, Minder C: Bias in meta-analysis detected by a simple, graphical test. BMJ 1997, 315:629-634.

30. Ji LD: Clinical report about interruption of intrauterine transmission of hepatitis B virus infection with HBIG. Modern Preventive Med 2003, 30:380

31. $X u Q$, Xiao L, Lu XB, Zhang $Y X$, Cai $X$ : A randomized controlled clinical trial: interruption of intrauterine transmission of hepatitis $B$ virus infection with HBIG. World J Gastroenterol 2006, 12:3434-3437.

32. Xu Q, Xiao L, Cai X, Zhang YX, Li F, Xu LS: A study of reducing the transmission of $\mathrm{HBV}$ from mother to infant in $\mathrm{HBeAg}$ positive pregnant women. Chin J Evidence-based Med 2004, 5:306-309.

33. Chen WL, Lu CS, Lai LP: Observation on the results of HBIG combined with hepatitis B vaccine interruption of vertical transmission of HBV. Chin Trop Med 2007, 7:1177-1178.

34. Sun B, Zheng RS, Yu SJ, Liu XL: The efficacy of hepatitis B combined with $\mathrm{HBIG}$ in infants born to $\mathrm{HBsAg}$ and $\mathrm{HBeAg}$ carrier mothers. Chin J of PHM 2007, 23:86-87. 
35. Wang YL, LV SF: A clinical study of interruptive effects of HBV specific immunoglobulins on the $\mathrm{HBsAg}+$ and $\mathrm{HBeAg}+$ women in midtrimester pregnancy. Med J Qilu 2007, 22:154-155.

36. Yan KH, Li LL, Chen SY, Wu M, Zhu MY, He LL: Assessment of effect of HBIG combined with hepatitis $B$ vaccine in interrupting vertical transmission of HBV from mothers to babies. Chin Trop Med 2009, 9:505-506.

37. Cui HY, Zhao CM, Shen XX: The efficacy of hepatitis B combined with $\mathrm{HBIG}$ in infants born to HBsAg and HBeAg carrier mothers. Chin J Dis Control Prev 2011, 15:545

38. Zhu Q, Lu Q, Gu X, Xu H, Duan S: A preliminary study on interruption of HBV transmission in uterus. Chin Med J (Engl) 1997, 110:145-147.

39. Zhu Q, Lv Q, Gu XH, Duan SC, Xu HF, Zhang XZ: A preliminary study on interruption of HBV transmission in uterus. Chin J Pediatr 1995, 33(2):93-95.

40. Jia $Q Q$, Gu Y: The blocking effect of hepatitis B immunoglobulin on HBV intrauterine infection and its indication. Chin J Neonatol 2001, 16:196-197.

41. Chi MZ, Wang YY, Shuai CX: Clinical study about interruption of intrauterine transmission of hepatitis $B$ virus infection with HBIG. Chin J Contemp Pediatr 2002, 4:127-128.

42. Chen $X Y$, Luo ZY, Xuan ZB, Yu LP: Study on immunoglobulin of hepatitis $B$ in stopping transmission from motherto infant. Zhejiang Prev Med 2003, 15:10-11.

43. Han GR, Yu MM, Shen L, Tang X, Wu MM, Zhang XY: Clinical study of interruption of intrauterine transmission of hepatitis $B$ virus infection with HBIG. Jiangsu Med J 2003, 29:833-835.

44. Han GR, Yu MM, Shen L, Tang YR, Wu MM, Zhang XY: Clinical study of interrupting $\mathrm{HBV}$ intrauterine infection by immunization of pregnant women and infant. Chin J Perinat Med 2004, 7:8-10.

45. Xing QX, Yue F, Zhang CH, Zong XY, Li ZZ, Wang JJ, Qi HY: Clinical study of interrupting $\mathrm{HBV}$ intrauterine infection by immunization of pregnant women with HBIG. J Appl Clin Pediatr 2003, 18:283-285.

46. Yue F, Xing QX, Li DR, Deng TM: Clinical observation of blocking HBV intrauterine infection by injection of hepatitisB immuniglobulin before laboring. Clin Med Chin 2003, 19:1049-1050.

47. Zhu Q, Yu G, Yu H, Lv Q, Gu XH, Dong ZQ, Zhang X: A randomized control trial on interruption of HBV transmission in uterus. Chin Med J (Engl) 2003, 116:685-687.

48. Zhu QR, Yu GJ, Lv Q, Yu H, Gu XH, Zhang XZ: A randomized control trial on interruption of HBV transmission in uterus. Chin J Pediatr 2002, 40(8):478-480.

49. Chen $\mathrm{QM}$, Chen $\mathrm{W}$ : The clinical observation on the effect of hepatitis $B$ immunoglobulin on the interruption of hepatitis $B$ virus intrauterine infection. Hebei Med 2006, 12:534-536.

50. Yang LT, Nie Y: Clinical study of interrupting HBV intrauterine infection by immunization of pregnant women with HBIG. Ninth Infectious Diseases of National Science Conference Proceedings. Chinese Medical Association 2006, C116:268-269.

51. Yu H, Zhu QR, Chen SQ, Xie XB, Chen H, Wang JS, Wang XH, Dong ZQ, Fei $L E$, Zhang $X Z$ : Study on a antepartum immunoprophylaxis to interrupt the transmission of hepatitis B virus from mother to infant. Chin J Infect Dis 2006, 24(6):390-395.

52. Ji XH: The study on HBIG and vaccine to interrupt the HBV transmission from mother to infant. Shanghai J Pre Med 2007, 19:217.

53. LiU JY, LU GR: Comparison of efficiency of two methods in preventing HBV maternal-fetal transmission. Clin Med 2007, 27:13-14.

54. Wang FY, Lin $P$, Zhang $H Z$ : A randomized controlled trial on effect of hepatitis $B$ immune globulin in preventing hepatitis $B$ virus transmission from mothers to infants. Chin J Pediatr 2008, 5:61-63.

55. Zhao SK, LV HY: Effect of hepatitis $B$ vaccine combined with hepatitis $B$ immuglobulin in interruption of vertical transmission of HBV. J Pract Diag Ther 2008, 22:236-237.

56. Liu LZ, Zheng JS, Li Q, Yu XH, Deng W: The study of the significance infection of hepatitis $B$ virus of Pergn placenta and fetal in pregnant woman with hepatitis B surface antigen, ant-HBe and Anti-HBc positive. Acta Academiae Med Jiangxi 2009, 49(2):92-95.

57. Yuan GP, Li MZ, Yu K, Chu GF: Effect of recombinant hepatitis B vaccine combined with hepatitis $B$ immuglobulin in interruption of vertical transmission of HBV. Chin J Public Health 2009, 12:1479-1480.

58. Li WG, Zhang JL, Wang HQ: Hepatitis B infection among babies born by pregnant women with different $\mathrm{HBV}$ infection status by passive immunization. Modern Pre Med 2013, 4:633-634.
59. Lo KJ, Tsai YT, Lee SD, Yeh CL, Wang JY, Chiang BN, Wu TC, Yeh PS, Goudeau A, Coursaget P: Combined passive and active immunization for interruption of perinatal transmission of hepatitis B virus in Taiwan. Hepatogastroenterology 1985, 32:65-68.

60. Lo KJ, Tsai YT, Lee SD, Wu TC, Wang JY, Chen GH, Yeh CL, Chiang BN, Yeh $\mathrm{SH}$, Goudeau A: Immunoprophylaxis of infection with hepatitis B virus in infants born to hepatitis B surface antigen-positive carrier mothers. J Infect Dis 1985, 152:817-822.

61. Goudeau A, Lo KJ, Coursaget P, Tong MJ, Yeh CL, Tsai YT, Lee JK, Wu TC, Yeh SH, Lee SD: Prevention of hepatitis B virus infection in children born to $\mathrm{HBsAg}$ positive/HBeAg positive mothers. Preliminary results of active and passive-active immunization. Dev Biol Stand 1983, 54:399-404.

62. Sha $\mathrm{QH}$ : The study on the combination of domestic vaccine and HBIG to interrupt HBV transmission from mother to infant. Guangdong Med 1985, 9:8-10.

63. Wu SY, Yang WC: A preliminary study to interrupt HBV transmission from mother to infant. Fujian Med J 1986, 1:15-16.

64. Farmer K, Gunn T, Woodfield DG: A combination of hepatitis B vaccine and immunoglobulin does not protect all infants born to hepatitis $B$ e antigen positive mothers. N Z Med J 1987, 100:412-414.

65. Theppisai U, Thanuntaseth C, Chiewsilp P, Siripoonya P: A comparison between the efficacy of passive-active and active immunization for prevention of perinatal transmission of hepatitis B virus. J Med Assoc Thai 1987, 70:459-462.

66. Ip HM, Lelie PN, Wong VC, Kuhns MC, Reesink HW: Prevention of hepatitis $B$ virus carrier state in infants according to maternal serum levels of HBV DNA. Lancet 1989, 1:406-410.

67. Ip HM, Wong VC, Lelie PN, Reesink HW, Schaasberg W, Yeung CY, Ma HK Hepatitis B infection in infants after neonatal immunization. Acta Paediatr Jpn 1989, 31:654-658.

68. Assateerawatt A, Tanphaichitr VS, Suvatte V, Yodthong S: Immunogenicity and efficacy of a recombinant DNA hepatitis B vaccine, GenHevac B Pasteur in high risk neonates, school children and healthy adults. Asian Pac J Allergy Immunol 1993, 11:85-91.

69. Li XX, Ye HQ, Luo JF, Du YX, Shi Q, Xiao LM, Yao FB, Xie GZ, Zhang R, Shi $H C$, Jiang QF, Wang LF: The study of interrupting HBV transmission from mother to infant using Hepatitis B vaccine and HBIG. Shanghai J Immunol 1994, 2:85-88.

70. Zhao HY, Wang SJ, Zheng XF: The study of interrupting HBV transmission from mother to infant using Hepatitis B vaccine and HBIG. Chin J Experment Clin Virol 1994, 4:352-354.

71. Kang P, Shen XM, Yu HM: Study on the efficacy of the genetic engineering vaccines against hepatitis $B$ for interruption of perinatal transmission. Chin J Nurse 1995, 7:390-392.

72. Poovorawan Y, Sanpavat S, Chumdermpadetsuk S, Safary A: Long-term hepatitis $B$ vaccine in infants born to hepatitis $B$ e antigen positive mothers. Arch Dis Child Fetal Neonatal Ed 1997, 77:F47-F51.

73. Poovorawan $Y$, Sanpavat S, Pongpunglert W, Safary A: Long term efficacy of hepatitis $B$ vaccine in infants born to hepatitis $B$ e antigen-positive mothers. Pediatr Infect Dis J 1992, 11:816-821.

74. Poovorawan Y, Sanpavat S, Pongpunlert W, Chumdermpadetsuk S, Sentrakula P, Chitinand S, Sakulramrung R, Tannirundorn Y: Comparison of a recombinant DNA hepatitis $B$ vaccine alone or in combination with hepatitis $B$ immune globulin for the prevention of perinatal acquisition of hepatitis B carriage. Vaccine 1990, 8(Suppl):S56-S59. S60-2.

75. Lin HF, Han XL, Meng ZD: Protective efficacy of recombinant HBsAg vaccine in prevention of mother-infant transmission of hepatitis $B$ virus. $J$ Hebei Med College Contin Edu 2000, 17:40-41.

76. Meng ZD, Han XL, Lin HF, Du M, Hou T, Yang CL, Dong YH, Zhao YL, Liu $\mathrm{HB}$ : Study on protective efficacy of $\mathrm{CHO}$ expressed recombinant $\mathrm{HBsAg}$ vaccine inblocking mother-infant transmission of hepatitis $B$ virus. Chin J Vaccine Immun 2001, 2:34-35.

77. Wang JS, Zhu QR: A long-term follow-up of hepatitis B vaccine inoculated and $\mathrm{HB}$ immunoglobulin combinely immunized infants born to $\mathrm{HBsAg}$ positive mothers. Chin J Vaccine Immun 2001, 2:96-99.

78. Wang JS, Zhu QR: A study on the efficacy of hepatitis B immunoglobulin combining with hepatitis $B$ vaccine in interrupting the maternal-infant transmission of hepatitis B virus. Chin J Pract Pediatr 2000, 9:558-560.

79. Sehgal A, Gupta I, Sehgal R, Ganguly NK: Hepatitis B vaccine alone or in combination with anti-HBs immunoglobulin in the perinatal prophylaxis of babies born to HBsAg carrier mothers. Acta Virol 1992, 36:359-366. 
80. Sehgal A, Sehgal R, Gupta I, Bhakoo ON, Ganguly NK: Use of hepatitis B vaccine alone or in combination with hepatitis $B$ immunoglobulin for immunoprophylaxis of perinatal hepatitis B infection. J Trop Pediatr 1992, 38:247-251.

81. Chen HL, Lin LH, Hu FC, Lee JT, Lin WT, Yang YJ, Huang FC, Wu SF, Chen SC, Wen WH, Chu CH, Ni YH, Hsu HY, Tsai PL, Chiang CL, Shyu MK, Lee PI, Chang FY, Chang MH: Effects of maternal screening and universal immunization to prevent mother-to-infant transmission of HBV. Gastroenterology 2012, 142:773-781.

82. Yin $Y$, Wu L, Zhang J, Zhou J, Zhang P, Hou H: Identification of risk factors associated with immunoprophylaxis failure to prevent the vertical transmission of hepatitis B virus. J Infect 2013, 66:447-452.

83. Wen WH, Chang MH, Zhao LL, Ni YH, Hsu HY, Wu JF, Chen PJ, Chen DS, Chen HL: Mother-to-infant transmission of hepatitis B virus infection: significance of maternal viral load and strategies for intervention. J Hepatol 2013, 59:24-30.

\section{Submit your next manuscript to BioMed Central and take full advantage of:}

- Convenient online submission

- Thorough peer review

- No space constraints or color figure charges

- Immediate publication on acceptance

- Inclusion in PubMed, CAS, Scopus and Google Scholar

- Research which is freely available for redistribution 